ARTICLE

DOI: $10.1038 / \mathrm{s} 41467-017-02697-5 \quad$ OPEN

\title{
GWAS of epigenetic aging rates in blood reveals a critical role for TERT
}

Ake T. Lu et al.\#

DNA methylation age is an accurate biomarker of chronological age and predicts lifespan, but its underlying molecular mechanisms are unknown. In this genome-wide association study of 9907 individuals, we find gene variants mapping to five loci associated with intrinsic epigenetic age acceleration (IEAA) and gene variants in three loci associated with extrinsic epigenetic age acceleration (EEAA). Mendelian randomization analysis suggests causal influences of menarche and menopause on IEAA and lipoproteins on IEAA and EEAA. Variants associated with longer leukocyte telomere length $(L T L)$ in the telomerase reverse transcriptase gene (TERT) paradoxically confer higher IEAA $\left(P<2.7 \times 10^{-11}\right)$. Causal modeling indicates TERT-specific and independent effects on LTL and IEAA. Experimental hTERTexpression in primary human fibroblasts engenders a linear increase in DNA methylation age with cell population doubling number. Together, these findings indicate a critical role for hTERT in regulating the epigenetic clock, in addition to its established role of compensating for cell replication-dependent telomere shortening. 
$\mathrm{D}$ NA methylation (DNAm) profiles of sets of cytosine phosphate guanines (CpGs) allow one to develop accurate estimators of chronological age which are referred to as "DNAm age", "epigenetic age", or the "epigenetic clock". Across the life course the correlation between DNAm age and chronological age is $>0.95^{1,2}$. Individuals whose leukocyte DNAm age is older than their chronological age ("epigenetic age acceleration") display a higher risk of all-cause mortality after accounting for known risk factors ${ }^{3-6}$, and offspring of centenarians exhibit a younger DNAm age ${ }^{7}$. Epigenetic age acceleration in blood is associated with cognitive impairment, neuro-pathology in the elderly ${ }^{8,9}$, Down syndrome ${ }^{10}$, Werner syndrome $^{11}$, Parkinson's disease ${ }^{12}$, obesity ${ }^{13}$, HIV infection ${ }^{14}$, and frailty ${ }^{15}$, menopause ${ }^{16}$ but it only displays weak correlations with clinical biomarkers ${ }^{17,18}$. DNAm age shows no apparent correlation with leukocyte telomere length (LTL), whose pace of shortening in cultured somatic cells has been referred to as the 'mitotic clock'. In vivo, DNAm age and telomere length appear to be independent predictors of mortality ${ }^{19}$.

Here we examine two widely used measures of epigenetic age acceleration: (a) intrinsic epigenetic age acceleration (IEAA), based on 353 CpGs described by Horvath ${ }^{2}$, which is independent of age-related changes in blood cell composition, and (b) extrinsic epigenetic age acceleration (EEAA), an enhanced version of that based on 71 CpGs described by Hannum (2013) which upweights the contribution of blood cell count measures ${ }^{1,6}$. IEAA and EEAA are only moderately correlated $(r=0.37)$. IEAA measures cell-intrinsic methylation changes, exhibits greater consistency across different tissues, appears unrelated to lifestyle factors and probably indicates a fundamental cell aging process that is largely conserved across cell types ${ }^{2,6}$. By contrast, EEAA captures age-related changes in leukocyte composition and correlates with lifestyle and health-span related characteristics, yielding a stronger predictor of all-cause mortality ${ }^{6,18}$. To dissect the genetic architecture underlying DNAm age of blood, we performed genome-wide association studies (GWAS) of IEAA and EEAA based on leukocyte DNA samples from almost 10,000 individuals. Our GWAS identifies a total of five loci associated with IEAA and three loci associated with EEAA at genome-wide significance. One of the loci associated with IEAA co-locates with the Telomerase Reverse Transcriptase (TERT) gene on chromosome 5. Variants in TERT that are associated with increased IEAA are also associated with longer telomeres. Our in vitro experiments indicate that hTERT expression is required for DNAm aging in human primary fibroblast cells. Finally, our Mendelian randomization analyses reveal that age at menarche and age at menopause have a causal effect on IEAA.

\section{Results}

GWAS meta-analyses for IEAA and EEAA. Genomic analyses were performed in 9907 individuals (aged 10-98 years), from 15 data sets, adjusted for chronological age and sex (Supplementary Table 1, Fig. 1, and Supplementary Note 1). Eleven data sets comprised individuals of European ancestry (84.7\%) and four comprised individuals of African (10.3\%) or Hispanic ancestry (5.0\%). GWAS genotypes were imputed to 7.4 million variants using the 1000 genomes reference panel. To estimate the heritability of epigenetic age acceleration in blood, we used both pedigree- and SNP-based methods (Methods section). Our pedigree-based estimates of heritability were $h_{\text {IEAA }}^{2}=0.37$ and $h_{\text {EEAA }}^{2}=0.33$ in individuals of European ancestry, which is consistent with previous heritability estimates in twins ${ }^{2}$ and with those obtained in other tissues (e.g., adipose and brain) ${ }^{9,13,20}$. SNP-based estimates of heritability in our European ancestry cohorts were lower, $h_{\mathrm{IEAA}}^{2}=0.19$ and $h_{\mathrm{EEAA}}^{2}=0.19$ (Supplementary Table 2).

We first performed GWAS meta-analysis of IEAA and EEAA only in our European ancestry cohorts $(N=8393)$. Variants with suggestive associations $\left(P<1.0 \times 10^{-5}\right)$ were then evaluated in non-European ancestry cohorts $(N=1514)$, followed by a combined meta-analysis across the two strata (Fig. 1a, b). We found no evidence for genomic inflation in individual studies $\left(\lambda_{\mathrm{GC}}=0.99 \sim 1.06\right.$, Supplementary Tables 3 and 4$)$ or in the European ancestry meta-analysis $\left(\lambda_{\mathrm{GC}}=1.03 \sim 1.05 ; \mathrm{LD}\right.$ score regression (LDSC) intercept terms $\beta_{0, \text { IEAA }}=1.004$ and $\beta_{0, \mathrm{EEAA}}=1.004$; Supplementary Fig. 1 and Supplementary Table 2). Variant associations that were genome-wide significant $\left(P<5.0 \times 10^{-8}\right)$ according to the fixed-effect metaanalysis were also evaluated with the trans-ethnic heterogeneity test that is implemented in the MANTRA software ${ }^{2 \mathrm{P}}$. The MANTRA software provides a meta-analysis estimate, known as Bayes Factor (BF), that is arguably more stringent than the fixedeffects meta-analysis because it accounts for genetic heterogeneity between different study populations. Our study focused on genetic variants that met two criteria: fixed effects meta $P<5.0 \times 10^{-8}$ and MANTRA $\log _{10} \mathrm{BF} \geq 6\left(\cong P<5 \times 10^{-8}\right)$.

For IEAA, we identified 264 associated variants, mapping to five genomic loci (3q25.33, 5p15.33, 6p22.3, 6p22.2, and 17q22, Table 1, Supplementary Data 1, Fig. 2, and Supplementary Fig. 2). Conditional analyses revealed a secondary signal for IEAA at 6p22.3 (Table 1, Supplementary Figs. $3 \mathrm{c}$ and h). For EEAA, we identified 440 associated variants, mapping to three loci (4p16.3, 10p11.1, and 10p11.21; Table 1, Supplementary Data 1, Fig. 2, and Supplementary Fig. 4); however, the two lead SNPs, rs71007656 and rs1005277 at 10p11.1 and 10p11.21, respectively, are moderately correlated $\left(r_{\mathrm{EUR}}^{2}=0.35\right.$, Table 1$)$. Conditional analysis showed that the association of the INDEL variant rs71007656 partially derived from its association with rs 1005277 because the $P$-value of rs71007656 was no longer genome-wide significant in a conditional model of rs1005277. By contrast, the SNP rs 1005277 remained genome-wide significant in a conditional model of rs71007656 (Supplementary Fig. 5b, c and e, f). Associations were consistent across studies (Supplementary Figs. 6 and 7), except for one locus (6p22.3: Cochran's $I^{2}=58 \%$, MANTRA posterior probability of heterogeneity $=0.64$, Table 1 and Supplementary Data 1). At each of the five IEAA related loci, the risk alleles conferred between 0.41 and 1.68 years higher IEAA (Table 1). Of the five loci associated with IEAA, four also exhibited at least suggestive and sign-consistent associations with EEAA (most significant $P=6.6 \times 10^{-7}$, Supplementary Data 2. By contrast, SNPs in the three loci associated with EEAA were not associated $(P>0.05)$ with IEAA (Supplementary Data 2). Analysis of published chromatin state marks ${ }^{22}$ showed that most lead variants are in chromosomal regions that are transcribed in multiple cell lines (Supplementary Fig. 8). Two loci, 6p22.2 and 6p22.3, colocate (within $1 \mathrm{Mb}$ ) with $\mathrm{CpGs}$ that contribute to the Horvath estimate of DNAm age (Table 1 and Supplementary Data 1), and it is possible that these genotypic associations with IEAA arise from direct SNP effects on local methylation (Supplementary Note 2 and Supplementary Figs. 9 and 10).

Transcriptomic studies in leukocytes. To learn about potential functional consequences of these associations, we conducted cis-eQTL analysis for each locus associated with IEAA or EEAA, using data on leukocyte mRNA expression in up to 15,295 samples from five studies (Fig. 1c; Methods section). We identified 11 putative cis-eQTLs located in seven of the eight associated loci (Supplementary Data 3). Each putative cis-eQTL was then analyzed by summary data-based Mendelian randomization 
a

Study data sets

b

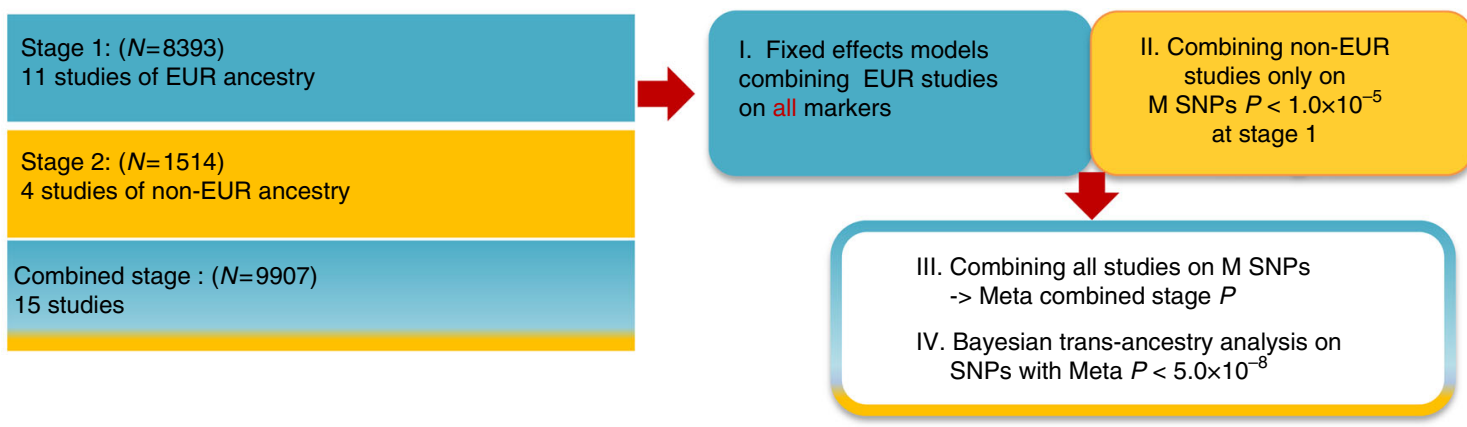

Genome-wide significant SNPs : Meta $P<5.0 \times 10^{-8} \& \log _{10} \mathrm{BF} \geq 6$

C Transcriptomic studies in blood $(N=15,295)$

$\begin{array}{lrllll}\text { Study } & N & \text { I. Blood cis-eQTL } & \text { SNPS } & \text { GE } \\ \text { 1. FHS } & 5257 & & \text { GE } & \text { IEAA/EEAA } & \text { GE } \\ \text { 2. GTEx } & 338 & \text { II. SMR analysis } & \text { cis SNPs } & \text { cis SNPs } \\ \text { 3. LSMeta... } & 5331 & & \text { IEAAEEAA }\end{array}$

d GWAS overlap of IEAA and LTL $(N=117,047)$ in TERT locus

I. LTL-SNPs co-associated with IEAA in $\operatorname{TERT}\left(P<5.0 \times 10^{-8}\right)$ and other 2 loci $(P<0.05)$

II. However, MR-Egger implies no causality.

III. SMR identifies pleiotropic association in TERT

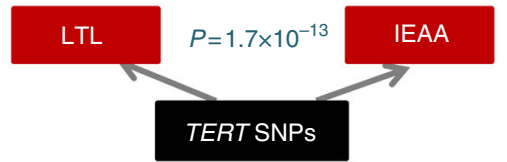

Paradoxical finding: alleles associated with longer telomeres are associated with higher IEAA

\section{e In vitro hTERT in human primary cells}

I. In vitro expressing hTERT and empty vector (control) in human fibroblasts.

II. Comparing the growth curve between hTERT and control for cell proliferations.

III. Horvath DNAm age increasing with days in culture in hTERT cells.

Trade-off: implied in between telomere length and DNAm age

\section{f MAGENTA gene set enrichment analysis in KEGG, GO and PANTHER}

g Examining genetic relationship with other 23 traits.

I. LDSC genetic correlation $\left(r_{g}\right)$ analysis for all the 23 traits.

II. MR-egger regression for elucidating determinants of epigenetic age acceleration.

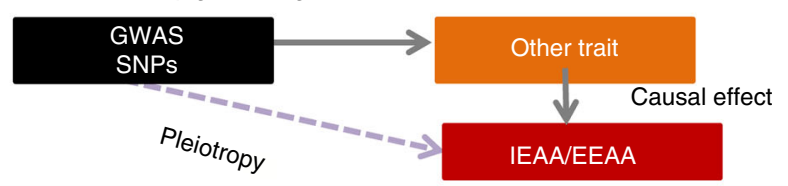

Fig. 1 Roadmap for studying genetic variants associated with epigenetic age acceleration in blood. The roadmap depicts our analytical procedures. a The study sets were divided into two stages according to European (EUR) and non-European ancestry. b Stage 1 yielded GWAS summary data on all QC SNPs and the combined stage yielded GWAS summary data on the SNPs with Meta EUR $P<1.0 \times 10^{-5}$ at stage 1 . Genome-wide significant loci were determined based on the association results from the combined stage. c Describes our transcriptomic studies: (I) blood cis-eQTL to identify potential functional genes, (II) summary statistics based Mendelian randomization (SMR) to assess the causal associations between expression levels and IEAA (or EEAA). d Describes our detailed analysis in the TERT locus, which was implicated by our GWAS of IEAA. Bidirectional Mendelian randomization via MR-Egger analysis did not reveal a direct causal effect between leukocyte telomere length and IEAA. Our in vitro studies validate our genetic findings by demonstrating that hTERT over-expression promotes epigenetic aging in e. To explore molecular pathways underlying epigenetic age acceleration, we conducted gene set enrichment analysis, as listed in f. Finally, we performed LDSC genetic correlation between IEAA or EEAA and a broad category of complex traits, followed by MR-Egger regression analysis, as depicted in $\mathbf{g}$. Abbreviations: GE = gene expression, LTL = leukocyte telomere length 
Table 1 Meta-analysis of GWAS of epigenetic age acceleration in blood

\begin{tabular}{|c|c|c|c|c|c|c|c|c|c|c|c|c|}
\hline \multirow[b]{2}{*}{ Band } & \multirow[b]{2}{*}{$\mathbf{N}_{\text {GWAS }}$} & \multirow[b]{2}{*}{ SNP } & \multirow[b]{2}{*}{ Gene } & \multirow[b]{2}{*}{ Mb } & \multirow[b]{2}{*}{ A1/A2 } & \multirow[b]{2}{*}{ MAF } & \multicolumn{3}{|c|}{ Fixed-effects } & \multicolumn{3}{|c|}{ Trans-ethnic } \\
\hline & & & & & & & Stage & Beta (SE) & P-value & $\boldsymbol{I}^{2}$ & $\log _{10} B F$ & $\boldsymbol{P}_{\text {HET. }}$ \\
\hline \multicolumn{13}{|l|}{ IEAA } \\
\hline \multirow[t]{3}{*}{$3 q 25.33$} & 23 & rs11706810 & TRIM59 & 160.2 & $\mathrm{C} / \mathrm{T}$ & 0.45 & EUR & $0.40(0.07)$ & $2.8 \times 10^{-8}$ & & & \\
\hline & & & & & & & Non-EUR & $0.44(0.19)$ & $1.8 \times 10^{-2}$ & & & \\
\hline & & & & & & & Combined & $0.41(0.07)$ & $1.6 \times 10^{-9}$ & $3 \%$ & 7.5 & 0.26 \\
\hline \multirow[t]{3}{*}{ 5p15.33 } & 11 & rs2736099 & TERT & 1.3 & $A / G$ & 0.36 & EUR & $0.64(0.09)$ & $4.7 \times 10^{-12}$ & & & \\
\hline & & & & & & & Non-EUR & $0.50(0.30)$ & $9.9 \times 10^{-2}$ & & & \\
\hline & & & & & & & Combined & $0.63(0.09)$ & $1.3 \times 10^{-12}$ & $0 \%$ & 10.6 & 0.47 \\
\hline \multirow[t]{6}{*}{$6 p 22.3$} & 104 & rs143093668 & KIF13A-NHLRC1 & 18.1 & $\mathrm{~T} / \mathrm{C}$ & 0.05 & EUR & $-1.78(0.10)$ & $1.9 \times 10^{-21}$ & & & \\
\hline & & & & & & & Non-EUR & $-1.37(0.33)$ & $2.4 \times 10^{-5}$ & & & \\
\hline & & & & & & & Combined & $-1.68(0.16)$ & $4.2 \times 10^{-25}$ & $58 \%$ & 23.1 & 0.64 \\
\hline & & rs6915893 & KIF13A-NHLRC1 & 18.1 & $\mathrm{~T} / \mathrm{C}$ & 0.39 & EUR & $0.56(0.08)$ & $5.1 \times 10^{-13}$ & & & \\
\hline & & & & & & & Non-EUR & $0.33(0.17)$ & $5.8 \times 10^{-2}$ & & & \\
\hline & & & & & & & Combined & $0.52(0.07)$ & $1.6 \times 10^{-13}$ & $27 \%$ & 11.4 & 0.34 \\
\hline \multirow[t]{3}{*}{$6 p 22.2$} & 108 & rs73397619d & LRRC16A-SCGN & 25.6 & $\mathrm{C} / \mathrm{T}$ & 0.29 & EUR & $-0.46(0.08)$ & $5.9 \times 10^{-9}$ & & & \\
\hline & & & & & & & Non-EUR & $-0.46(0.18)$ & $1.2 \times 10^{-2}$ & & & \\
\hline & & & & & & & Combined & $-0.46(0.03)$ & $2.3 \times 10^{-10}$ & $18 \%$ & 8.3 & 0.32 \\
\hline \multirow[t]{3}{*}{$17 q 22$} & 18 & rs78781855 & STXBP4 & 53.1 & $\mathrm{G} / \mathrm{T}$ & 0.22 & EUR & $-0.42(0.09)$ & $1.6 \times 10^{-6}$ & & & \\
\hline & & & & & & & Non-EUR & $-0.88(0.23)$ & $1.5 \times 10^{-4}$ & & & \\
\hline & & & & & & & Combined & $-0.47(0.08)$ & $5.6 \times 10^{-9}$ & $26 \%$ & 7.2 & 0.45 \\
\hline \multicolumn{13}{|l|}{ EEAA } \\
\hline \multirow[t]{3}{*}{ 4p16.3 } & 59 & rs10937913 & TNIP2 & 2.8 & $A / G$ & 0.46 & EUR & $-0.60(0.01)$ & $3.8 \times 10^{-10}$ & & & \\
\hline & & & & & & & Non-EUR & $-0.56(0.23)$ & $1.4 \times 10^{-2}$ & & & \\
\hline & & & & & & & Combined & $-0.59(0.09)$ & $1.7 \times 10^{-11}$ & $0 \%$ & 9.2 & 0.24 \\
\hline \multirow[t]{3}{*}{ 10p11.21 } & 59 & rs71007656 & ANKRD30A-ZNF248 & 40.0 & $\mathrm{R} / \mathrm{I}^{\mathrm{e}}$ & 0.49 & EUR & $0.61(0.1-)$ & $1.1 \times 10^{-9}$ & & & \\
\hline & & & & & & & Non-EUR & $0.52(0.22)$ & $2.0 \times 10^{-2}$ & & & \\
\hline & & & & & & & Combined & $0.59(0.09)$ & $7.5 \times 10^{-11}$ & $0 \%$ & 8.5 & 0.29 \\
\hline \multirow[t]{3}{*}{ 10p11.1 } & 322 & rs1005277 & ZNF248-ZNF25 & 38.2 & $\mathrm{~A} / \mathrm{C}$ & 0.28 & EUR & $0.78(0.11)$ & $2.6 \times 10^{-13}$ & & & \\
\hline & & & & & & & Non-EUR & $0.45(0.29)$ & $1.1 \times 10^{-1}$ & & & \\
\hline & & & & & & & Combined & $0.74(0.10)$ & $1.2 \times 10^{-13}$ & $32 \%$ & 11.5 & 0.32 \\
\hline \multicolumn{13}{|c|}{ 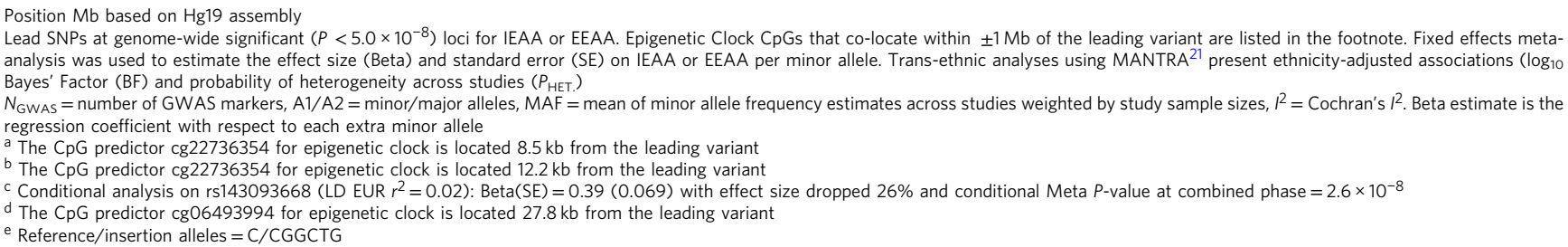 } \\
\hline
\end{tabular}

(SMR), which infers the association between gene transcript levels and the outcome trait ${ }^{23}$ (Methods section). Three transcripts were associated with IEAA: KPNA4 at 3q25.33, TPMT at 6p22.3 and STXBP4 at $17 \mathrm{q} 22$; and three transcripts were associated with EEAA: RNF4 at 4p16.3, ZNF25 at 10p.21, and HSD17B7P2 at 10p11.21 and 10p11.1 (Table 2 and Supplementary Table 5). Notably, STXBP4, encoding the syntaxin-binding protein, is a reported locus for age at menarche ${ }^{24}$, and our lead SNP for IEAA was also associated with age at menarche (rs78781855, $P=0.0002)$.

Paradoxical SNP association between IEAA and LTL in TERT. The TERT locus (in 5p15.33) harbored 11 genome-wide significant SNPs for IEAA but conditional analysis did not reveal any secondary signal (Table 1, Fig. 3a, and Supplementary Data 1). The leading SNP, rs2736099, was located in a region transcribed in human embryonic stem cells, induced pluripotent stem cells, and hematopoietic stem cells (Supplementary Fig. 8b), and each minor allele conferred 0.6 years higher IEAA $(P=1.3 \times$ $10^{-12}$; Table 1, Supplementary Fig. 6b). Our IEAA locus at TERT closely overlaps the reported GWAS locus for LTL ${ }^{25-27}$ (Fig. 3b). To further demonstrate that our GWAS findings surrounding TERT and IEAA are not mediated by LTL, we carried out an additional GWAS analysis of an LTL adjusted measure of IEAA in the subset $(n=785)$ of individuals for whom both IEAA and
LTL were available. As expected, the LTL adjusted measure of IEAA continued to exhibit significant associations with the variants in the TERT locus (Methods section and Supplementary Fig. 11).

SMR analysis indicated that the association signals for LTL and IEAA at this TERT locus share the same underlying causal variant (as indicated by a non-significant HEIDI test, Supplementary Table 6, Supplementary Fig. 12c). Intriguingly, TERT alleles associated with a longer LTL were robustly associated with increased IEAA $\left(P \sim 1.0 \times 10^{-11}\right.$, Table 3 , Supplementary Table 7 , and Supplementary Fig. 13).

Other known GWAS LTL signals (at 10q24.33 near OBFC1 and at 16q23.3 near MPHOSPH6), also exhibited modest associations with IEAA $\left(4.1 \times 10^{-3} \leq P \leq 3.7 \times 10^{-2}\right)$, but others, such as the gene TERC (on $3 \mathrm{q} 26.2$ ) encoding the telomerase RNA component, showed no association (Table 3). Using the lead variants for each trait in MR-Egger analyses ${ }^{28}$, we found pleiotropic effects specific to TERT on LTL and IEAA without evidence for a causal relationship between LTL and IEAA $(P=0.7)$, Table 4 and Supplementary Table 8 .

Blood cell composition vs. variants in TERT. By definition, IEAA is independent of various blood cell count estimates, i.e., it does not correlate with imputed blood cell abundance measures 
a IEAA

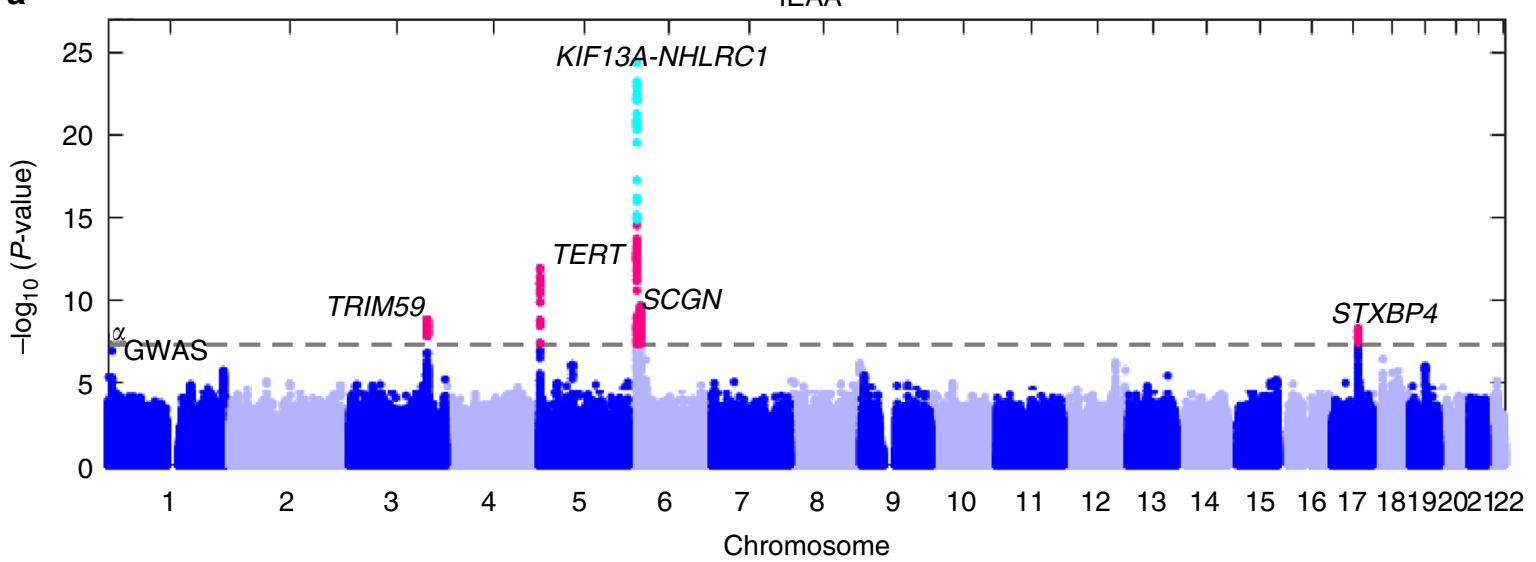

b

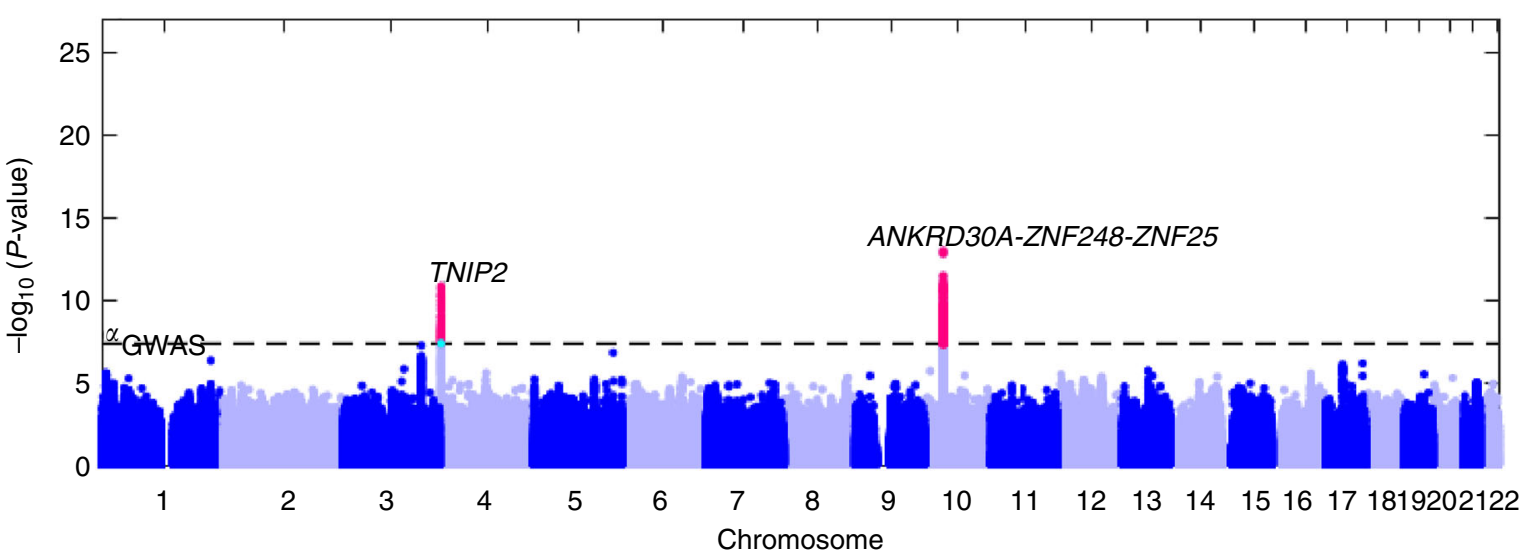

Fig. 2 Genome-wide meta-analysis for intrinsic and extrinsic age acceleration in blood. Manhattan plots for the meta-analysis $P$-values resulting from 15 studies comprised of 9907 individuals. The $y$-axis reports log transformed $P$-values for $\mathbf{a}$ intrinsic epigenetic age acceleration (IEAA) or $\mathbf{b}$ extrinsic epigenetic age acceleration (EEAA). The horizontal dashed line corresponds to the threshold of genome-wide significance $\left(P=5.0 \times 10^{-8}\right)$. Genome-wide significant common SNPs (MAF $\geq 5 \%)$ and low frequency SNPs $(2 \% \leq M A F<5 \%)$ are colored red and cyan, respectively

(Methods section). By contrast, LTL exhibits the expected positive correlation with the abundance of naive $\mathrm{CD}^{+} \mathrm{T}$ cells $\left(r=0.22, P=4.6 \times 10^{-10}\right)$ and naive $\mathrm{CD}^{+} \mathrm{T}$ cells $(r=0.13$, $P=0.00026)$ even after adjusting for chronological age (Supplementary Fig. 14). Consistent with our paradoxical association between LTL-related SNPs and IEAA, we find that TERT variants associated with a higher abundance of naive $\mathrm{T}$ cells (indicative of a younger adaptive immune system) are positively associated with higher values of IEAA and higher values of EEAA (indicative of an older epigenetic age, Supplementary Figs. 15 and 16, and Supplementary Data 4). However, none of the SNPs in the TERT locus was significantly associated with cell counts after correcting for multiple comparisons (uncorrected $P>0.003$, Supplementary Data 4) despite of the large sample size $N>5000$.

hTERT is required for DNAm aging in human primary cells. As we were unable to functionally link IEAA to TERT through cis-eQTL analysis at 5p15.33, we examined the effects of experimentally induced hTERT expression on IEAA in a primary human cell culture model. We introduced a TERT-expressing vector or empty vector (as control) into primary fibroblasts isolated from human neonatal foreskin. Transduced TERT-expressing and non-TERT cells were cultured in parallel. Upon reaching confluence, the cells were collected, counted, seeded into fresh plates, and profiled using the Illumina Infinium $450 \mathrm{~K}$ DNA methylation array.
While non-TERT cells senesced after $\sim 150$ days, TERTexpressing cells continued to proliferate unabated at a constant rate with time in culture (Fig. 3c). Single-time point analyses (Fig. 3d) showed that TERT-expressing cells exhibited a linear relationship between time in culture and the Horvath estimate of DNAm age (equivalent to a DNAm age of 50 years at 150 days), whereas in non-TERT cells DNAm age plateaued (equivalent to a DNAm age of 13 years) in spite of continued proliferation to the point of replicative senescence. Notably, DNAm age did not increase in TERT-expressing cells that received regular media change but were not passaged throughout the entire observation period of 170 days (right most bar in Fig. 3d). These cells were not senescent, given that their subsequent passaging resulted in normal proliferation. In multivariable regression analysis, the associations of DNAm age with cell passage number and cell population doubling number were highly modified by TERTexpression ( $P$-interaction: $P=1.6 \times 10^{-6}$ and $P=4.0 \times 10^{-5}$, respectively; Supplementary Table 9). In the absence of TERTexpression, DNAm age did not increase with cell passage number, cell population doubling number, or time in culture.

Other putative determinants of epigenetic age acceleration. To systematically elucidate possible further biological processes that influence epigenetic age acceleration, we tested our full genomewide association statistics for IEAA and EEAA using a number of approaches. First, we used MAGENTA ${ }^{29}$ (Methods section) to 
Table 2 Summary of transcriptomic studies for loci associated with epigenetic age acceleration

\begin{tabular}{|c|c|c|c|c|c|c|}
\hline \multirow[b]{2}{*}{ Band } & \multirow[b]{2}{*}{ SNP } & \multirow{2}{*}{$\begin{array}{l}\text { Meta GWAS } \\
\text { P-value (sign) }\end{array}$} & \multicolumn{3}{|c|}{ cis-eQTL } & \multirow{2}{*}{$\begin{array}{l}\text { SMR } \\
\text { P-value (sign) }\end{array}$} \\
\hline & & & Database & Gene & P-value (sign) & \\
\hline \multicolumn{7}{|l|}{ IEAA } \\
\hline $3 q 25.33$ & rs11706810 & $1.6 \times 10^{-9}(+)$ & FHS & KPNA4 & $4.2 \times 10^{-23}(+)$ & $5.1 \times 10^{-5}(+)$ \\
\hline \multirow[t]{3}{*}{$6 p 22.3$} & rs6915893 & $1.6 \times 10^{-13}(+)$ & FHS & TPMT & $6.0 \times 10^{-14}(+)$ & $1.1 \times 10^{-7}(+)$ \\
\hline & & & GTEx & & $1.5 \times 10^{-4 \star}(+)$ & $2.2 \times 10^{-6}(+)$ \\
\hline & & & LSMeta & & $2.3 \times 10^{-27}(+)$ & $3.5 \times 10^{-7}(+)$ \\
\hline $17 q 22$ & rs78781855 & $5.6 \times 10^{-9}(-)$ & FHS & STXBP4 & $1.0 \times 10^{-88}(-)$ & $6.2 \times 10^{-4}(+)$ \\
\hline \multicolumn{7}{|l|}{$E E A A$} \\
\hline $4 p 16.3$ & rs2341303 & $6.5 \times 10^{-11}(-)$ & LSMeta & RNF4 & $1.6 \times 10^{-10}(-)$ & $2.0 \times 10^{-3}(+)$ \\
\hline \multirow[t]{2}{*}{ 10p11.21 } & rs71007656 & $7.5 \times 10^{-11}(+)$ & FHS & ZNF25 & $7.9 \times 10^{-7}(+)$ & $2.8 \times 10^{-3}(+)$ \\
\hline & & & GTEx & HSD17B7P2 & $3.0 \times 10^{-8}(+)$ & $6.9 \times 10^{-6}(+)^{b}$ \\
\hline 10p11.1 & rs1005277 & $1.2 \times 10^{-13}(+)$ & GTEx & HSD17B7P2 & $1.1 \times 10^{-5}(+)$ & $6.9 \times 10^{-6}(+)^{b}$ \\
\hline \multicolumn{7}{|c|}{$\begin{array}{l}\text { The table presents a total of six cis genes highlighted from transcriptomic study using three large-scale databases }(N=10,906) \text {, including }(1) \text { FHS }(N=5257),(2) \text { GTEx }(N=338) \text {, and (3) LSMeta (5311). } \\
\text { Each cis gene exhibited significant cis-eQTL with several nearby GWAS SNPs at FDR } q<0.05 \text { in at least one study and also showed a significant pleiotropic association in SMR analysis at } P<0.05 \text { after } \\
\text { Bonferroni correction. For each gene, we list the unadjusted } P \text {-values (sign) from Meta GWAS for IEAA (or EEAA), cis-eQTL and SMR analysis. The column (sign) indicates the sign of } Z \text {-statistic at each } \\
\text { test while the test alleles were converted to the same alleles (with minor variants) for both Meta GWAS and cis-eQTL tests. The summary statistics of Meta GWAS and cis-eQTL are both based on the } \\
\text { leading marker with the most significant } P \text {-value in a given locus, according to the GWAS results } \\
\text { ^ FDR }>0.05 \text { but FDR }<0.05 \text { associated with other GWAS SNPs (Supplementary Data } 3 \text { ) } \\
\text { a The SNP rs2341303 is a surrogate of the leading marker rs } 10937913 \text { in } 4 \text { p16.3 (LD } r_{\text {EUR }}^{2}=0.98 \text { ) } \\
\text { b The SMR results are derived from the same model as the analysis used the cis SNPs of the gene HSD17B7P2 }\end{array}$} \\
\hline
\end{tabular}

identify biological pathways that are enriched for genes that harbor associated variants. For IEAA, nuclear transport $(\mathrm{FDR}=$ $0.017), \mathrm{Fc}$ epsilon RI signaling $(\mathrm{FDR}=0.027)$, and colorectal cancer processes $(\mathrm{FDR}=0.042)$ were implicated. For EEAA, mRNA elongation $(\mathrm{FDR}=0.011)$, mRNA transcription $(\mathrm{FDR}=$ $0.018)$, and neurotrophin signaling pathway $(F D R=0.042)$ were implicated (Supplementary Table 10). The GO gene set involved in telomere maintenance showed nominally significant enrichment with IEAA $(P=0.04$, Supplementary Table 11$)$, which is consistent with our results surrounding the overlap between IEAA and telomere length associated genes.

Second, we explored the genetic correlations $\left(r_{\mathrm{g}}\right)$ between IEAA or EEAA and 27 phenotypes using LD score regression analysis of summary level GWAS data ${ }^{30}$ (Methods section and Fig. 1g). We observed moderate positive genetic correlation between IEAA and EEAA $\left(r_{\mathrm{g}}=0.5, P_{r_{\mathrm{g}}}=8.9 \times 10^{-3}\right)$. IEAA showed weak positive genetic correlations with central adiposity (waist circumference) and metabolic disease-related traits, and EEAA showed stronger positive genetic correlations with central adiposity $\left(r_{\mathrm{g}}=0.15\right.$ with waist circumference, $P=9.0 \times 10^{-4}$ ) and metabolic disease-related traits (Table 4 and Supplementary Table 12). IEAA and EEAA also showed modest inverse genetic correlations with age at menopause.

Further, we performed a genetic correlation analysis using all available GWAS summary data from the LD Hub platform ${ }^{31}$ (see URL), identifying additional traits at nominal significance levels $(P<0.05)$. Lung function measures (forced expiratory volume) and brain volume measures exhibited negative genetic correlations with both IEAA and EEAA (Supplementary Data 5). Further, IEAA exhibited a positive genetic correlation $r_{\mathrm{g}}$ with primary sclerosing cholangitis and a negative genetic correlation with father's age at death.

Third, we performed MAGENTA based hypergeometric analyses to test whether the top 2.5 and $10 \%$ of genes enriched for GWAS associations with IEAA or EEAA overlap with the top enriched genes for a range of complex traits (Methods section). This analysis suggested several additional possible genetic overlaps, including Huntington disease onset ${ }^{32}$ and bipolar disorder with IEAA, schizophrenia with EEAA, and age-related demen$\operatorname{tia}^{20}$ with both IEAA and EEAA (Supplementary Data 6).

Finally, all the study traits were tested using MR-Egger regression which, by modeling the reported top genetic signals for each candidate trait, estimates the likely causal influence of that trait on IEAA or EEAA ${ }^{28}$ (Methods section). Nominally significant causal relationships on higher IEAA and EEAA were found for low-density lipoprotein (LDL) and total cholesterol levels $(P<0.05$, Supplementary Tables 13 and 14) and for triglyceride levels on IEAA $\left(P=3.0 \times 10^{-2}\right.$, Table 4). Earlier menarche and menopause were associated with higher IEAA; each 1-year earlier age at menarche was associated +1.03 years higher IEAA $\left(P=4.1 \times 10^{-3}\right)$ and each 1 -year earlier age at menopause was associated +0.43 years higher IEAA $(P=3.5 \times 10$ ${ }^{-3}$ ) (Table 4 ). A sensitivity analysis shows that the observed causal associations do not result from instrumental variable SNPs colocating with CpG sites from DNAm age estimators (Methods section and Supplementary Table 15). Our MR-Egger analysis revealed a causal effect of IEAA on a rough proxy of life span (father's age at death) $\left(P=1.9 \times 10^{-2}\right.$, Supplementary Table 16), which is consistent with previous studies that demonstrated that epigenetic age acceleration predicts life $\operatorname{span}^{3-7}$.

\section{Discussion}

This large genomic study provides several insights into the regulation of epigenetic aging, including apparently opposing roles for TERT on DNAm age and LTL. TERT encodes the catalytic subunit of telomerase, which counters telomere shortening during cell division $^{33}$. TERT also possesses activities unrelated to telomere maintenance, such as in DNA repair, cell survival, protection from apoptosis or necrosis, stimulation of growth ${ }^{34-36}$ and cell proliferation, possibly by decreasing p 21 production $^{37}$. Here we show an additional pleiotropic role of TERT on advancing cell intrinsic DNAm age during cell proliferation. Our findings provide an explanation for the previously reported rapid rate of DNAm aging during embryonic development and early postnatal life, which are stages of rapid organismal growth accompanied by high levels of TERT activity and cell division ${ }^{38-40}$.

The paradoxical finding that TERT alleles associated with longer telomeres are associated with higher IEAA is supported by (a) significant meta-analysis $P$-values, (b) validation in multiple large cohort studies, (c) in vitro support using primary fibroblasts, (d) consistent associations of other LTL associated genes (OBFC1 and MPHOSPH6), and (e) consistent genetic associations with select leukocyte subsets (e.g., naive T cells). While the paradoxical 


\section{a}

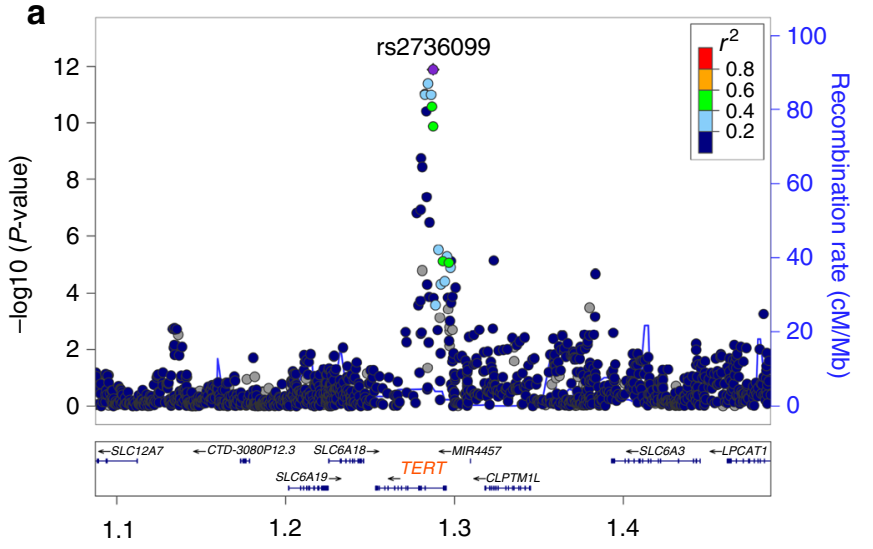

b

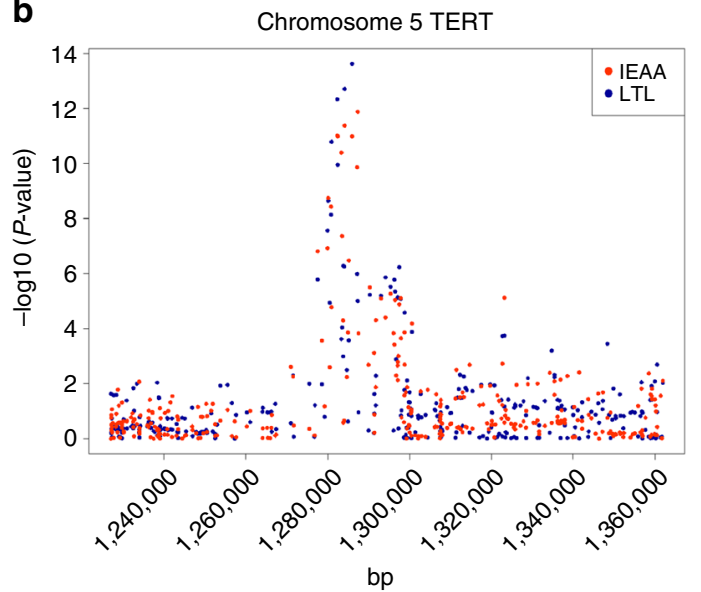

C

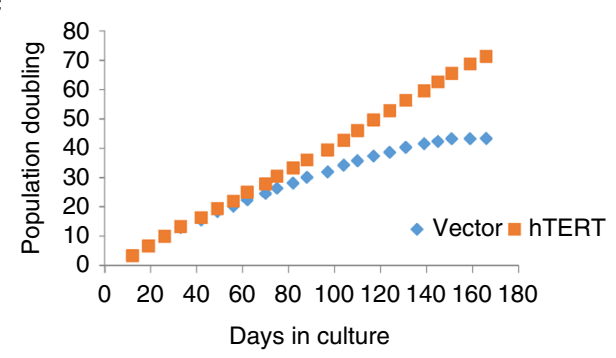

d

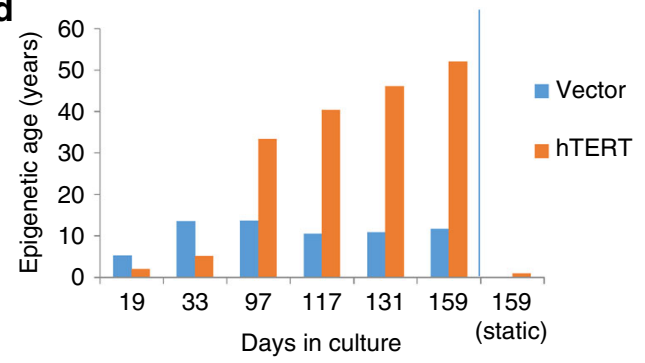

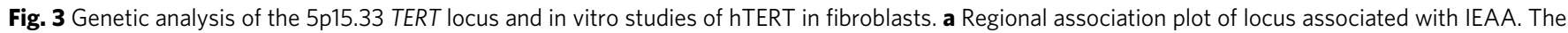
$y$-axis depicts log-transformed meta-analysis P-values across all studies 1-15. The colors visualize linkage disequilibrium (LD) $r^{2}$ between rs2736099 (colored in purple) and neighboring SNPs. b TERT-locus association with IEAA (marked in red) overlaid with the association with leukocyte telomere length (LTL) given by Bojesen et al. ${ }^{25}$ (marked in blue). Note that several SNPs in the TERT locus are associated with both IEAA and leukocyte telomere length at a genome-wide significant level. c Growth of human primary fibroblasts $(n=46)$ represented as population doublings ( $y$-axis) vs. days in culture

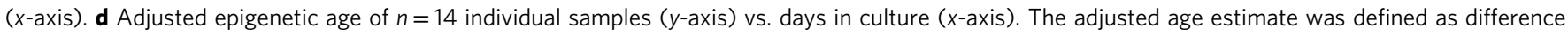
between Horvath DNAm age minus 28 years, since the former exhibited a substantial offset in fibroblasts. DNAm ages are increased in the hTERT

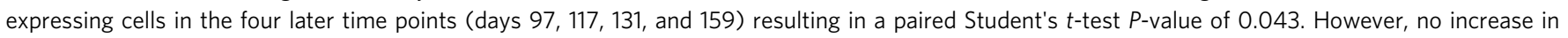
DNAm age can be observed in cells that are static (last bar)

finding cannot be disputed on scientific grounds, its biological interpretation remains to be elucidated. In our genetic studies, we found no evidence for a broader causal inter-relationship between telomere length and IEAA consistent with the lack of phenotypic association between these traits in our studies (WHI: $r=-0.05$, $P=0.16$; FHS: $r=0.0, \quad P=0.99$, ref. ${ }^{41}$ ) and in previous reports $^{15,19}$. The lack of a phenotypic association between LTL and IEAA indicates that the shared genetic influence (due to TERT and, to a lesser extent, OBFC1 and MPHOSPH6) is dwarfed by environmental influences acting on these traits.

While critically short telomere length is a well-established trigger of replicative senescence ${ }^{38,42-44}$, the functional consequences of epigenetic aging are yet unknown. Our experimental data suggest that epigenetic aging is not a determinant or marker of cell replicative senescence, since TERT-expressing cells continued to proliferate unabatedly despite well-advanced DNAm age, and non-TERT-expressing cells exhibited no DNAm age increase even at days 120-170 when proliferation had ceased and the cells had become senescent. Rather, TERT expression appears to allow cells to record their proliferation history. Our experiments show a clear association between DNAm age with cumulative population doubling (and days in culture) of cells with experimentally induced hTERT expression. This was not the case with proliferating cells that do not express hTERT. While the mechanism is yet to be elucidated, preliminary evidence suggests that DNAm age does indeed track the cell division of stem/progenitor cells, e.g., passage number is highly correlated with DNAm age of mesenchymal stem cells ${ }^{2,45}$.
Large-scale cross-sectional cohort studies have previously reported associations between epigenetic age acceleration (IEAA and EEAA) and body mass index and measures of insulin resistance ${ }^{18}$. Our genetic correlation analyses (between the measures of age acceleration and complex phenotypes) indicate that some of these associations may arise in part from shared genetic variants. The genetic correlation between IEAA and EEAA is quite high $\left(r_{\mathrm{g}}=0.52, P=0.0089\right)$ considering that these epigenetic biomarkers are based on distinct sets of $\mathrm{CpGs}$ and exhibit only modest phenotypic correlations $(r=0.37$ in the WHI and $r=0.36$ in the FHS). By contrast, IEAA/EEAA exhibit only relatively weak genetic correlations with many age related traits for a variety of reasons, including (i) technical variation/noise in the measures of epigenetic age acceleration, (ii) relatively low sample size in our GWAS of IEAA/EEAA, (iii) blood methylation data only provide insufficient information for capturing the dysfunction of other organ systems. The latter point is illustrated by the finding that obesity has a strong effect on the epigenetic age of liver tissue but only a weak effect on the epigenetic age of blood tissue $e^{13,18}$.

Our Mendelian randomization analyses provided tentative evidence for causal influences for blood lipid levels, but not for adiposity, on IEAA and EEAA. The suggestive causal effect of educational level on EEAA and the negative genetic correlation $\left(r=-0.13, P=1.0 \times 10^{-3}\right)$ are consistent with the previously observed phenotypic correlation between these traits ${ }^{18}$.

Finally, we found evidence for causal influences of earlier ages at menarche and menopause on higher IEAA. The directionally concordant influences of menarche and menopause, which signal 


\begin{tabular}{|c|c|c|c|c|c|c|c|c|c|c|c|}
\hline \multirow[t]{2}{*}{ Study } & \multirow[t]{2}{*}{ Chr } & \multirow[t]{2}{*}{ Gene } & \multirow[t]{2}{*}{ SNP } & \multirow[t]{2}{*}{ A1 } & \multirow[t]{2}{*}{ MAF } & \multicolumn{3}{|c|}{ Effect size $(\beta)$} & \multicolumn{3}{|c|}{ Meta P-value } \\
\hline & & & & & & LTL & IEAA & EEAA & LTL & IEAA & EEAA \\
\hline$\|$ & 2 & ACYP2 & rs11125529 & A & 0.14 & 0.06 & -0.06 & -0.15 & $4.5 \times 10^{-8}$ & 0.5 & $2.5 \times 10^{-1}$ \\
\hline III & 3 & PXK & rs6772228 & A & 0.06 & -120 & 0.26 & 0.20 & $3.9 \times 10^{-10}$ & $1.3 \times 10^{-1}$ & $3.7 \times 10^{-1}$ \\
\hline II & 4 & NAF1 & rs7675998 & A & 0.22 & -0.07 & -0.07 & 0.04 & $4.4 \times 10^{-16}$ & $4.4 \times 10^{-1}$ & 0.7 \\
\hline III & 5 & TERT & rs7726159 & A & 0.33 & 73 & 0.67 & 0.27 & $4.7 \times 10^{-17}$ & $\begin{array}{l}9.5 \times 10 \\
-12\end{array}$ & $6.4 \times 10^{-2}$ \\
\hline I & 5 & & rs7705526 & A & 0.33 & 0.51 & 0.61 & 0.18 & $2.3 \times 10^{-14}$ & $1.0 \times 10^{-11}$ & $1.9 \times 10^{-1}$ \\
\hline ॥ & 5 & & rs2736100 & C & 0.50 & 0.08 & 0.49 & 0.19 & $4.4 \times 10^{-19}$ & $2.7 \times 10^{-11}$ & $8.2 \times 10^{-2}$ \\
\hline III & 10 & OBFC1 & rs2487999 & $\mathrm{T}$ & 0.14 & 100 & 0.22 & 0.36 & $4.2 \times 10^{-14}$ & $3.7 \times 10^{-2}$ & $8.8 \times 10^{-3}$ \\
\hline ॥ & 10 & & rs9420907 & $\mathrm{C}$ & 0.20 & 0.07 & 0.26 & 0.20 & $6.9 \times 10^{-11}$ & $4.1 \times 10^{-3}$ & $9.9 \times 10^{-2}$ \\
\hline
\end{tabular}

P-values associated with age acceleration marked in italic if $<0.05$ and bold if $<5.0 \times 10^{-8}$

The table relates genome-wide significant association results of leukocyte telomere length (LTL) to two epigenetic age acceleration measures, IEAA and EEAA. We queried the results of 14 SNPs across 10 distinct susceptibility loci associated with LTL from three large-scale studies: (I) meta-analysis association of LTL in chromosome 5 TERT only $(N=53,724)^{25}$, (II) a genome-wide meta-analysis of LTL $(N=37,684)^{26}$, and $(I I I)$ a genome-wide meta-analysis of $L T L(N=26,089)^{27}$. Each row presents a genome-wide significant locus associated with $L T L$ in a given study, except chromosome 16 MPHOSPH6 and chromosome 20 BCL2L1 just slightly below genome-wide significance and highlighted by the corresponding studies as major findings. The listed markers are the leading SNPs with the most significant $P$-values associated with LTL at a given study and locus, sorted by chromosome, and position. Effect sizes of LTL association refer to the change in telomere lengths ( $\Delta T \mathrm{TL}$ ). Telomer engths were measured bas on the relative telomere to singe copy $(T / S)$ ratios using stand a scaling approaches applied to the measurements. The effect sizes for each age acceleration measure are in units of year

$\mathrm{Chr}=$ chromosome, $\mathrm{A} 1=$ reference allele, $\mathrm{MAF}=$ minor allele frequency; effect sizes corresponding additive models

the onset and cessation, respectively, of reproductive capacity, together with the lack of influence of any measure of adiposity on IEAA, suggest an effect of some yet identified driver of reproductive aging on DNAm aging. These findings, which suggest that sex steroids affect epigenetic aging, are consistent with previously reported associations regarding early menopause timing and higher IEAA in blood ${ }^{16}$. While menopausal hormone therapy was not found to be associated with IEAA in blood, it was found to be associated with younger epigenetic age acceleration in buccal epithelium $^{16}$. The effect of menopause is consistent with reported anti-aging effects of sex hormone therapy on buccal cells and the pro-aging effect of surgical ovariectomy in blood ${ }^{16}$. Early age at menarche, a widely studied marker of the timing of puberty in females, is associated with higher risks for diverse diseases of aging ${ }^{46}$. Our findings indicate epigenetic aging as a possible intrinsic mechanism that underlies the recently described link between menarche age-lowering alleles and shorter lifespan ${ }^{47}$.

\section{Methods}

GWAS Cohorts. GWAS meta-analysis was performed on 9907 individuals across 15 studies (Supplementary Table 1) coming from eight cohorts: Framingham Heart Study (FHS), TwinsUK, Women's Health Initiate (WHI), European Prospective Investigation into Cancer-Norfolk (EPIC-Norfolk), Baltimore Longitudinal Study of Aging (BLSA), Invecchiare in Chianti, aging in the Chianti Area Study (inCHIANTI), Brisbane Systems Genetics Study (BSGS), and Lothian Birth Cohorts of 1921 and 1936 (LBC) (Supplementary Note 1). Eleven data sets comprised individuals of European ancestry (EUR, 84.7\%) and four data sets comprised individuals of African ancestry (AFR, 10.3\%) or Hispanic ancestry (AMR, 5.0\%). Age range was $10-98$ years (69\% females).

DNA methylation age and measures of age acceleration. By contrasting the DNAm age estimate with chronological age, we defined measures of epigenetic age acceleration that are uncorrelated with chronological age. We evaluated two types of measures of epigenetic age acceleration in blood: cell-intrinsic and extrinsic epigenetic measures, which are independent of, or related to blood cell counts, respectively. Intrinsic epigenetic age acceleration (IEAA) is defined as the residual resulting from regressing the DNAm age estimate from Horvath (353 CpG markers) on chronological age and blood cell count estimates. By definition, IEAA does not depend on blood cell counts. By contrast, extrinsic epigenetic age acceleration (EEAA) depends on blood cell counts because it is defined by up-weighting the blood cell count contributions to the Hannum's epigenetic age estimator (71
CpGs). Thus, EEAA captures both age-related changes in blood cell types, as well as cell-intrinsic age-related changes in DNAm levels.

IEAA and EEAA are based on the DNAm age estimates from Horvath ${ }^{2}$ (353 CpG markers) and from Hannum et al. ${ }^{1}$ (71 CpGs), respectively. Mathematical details and software tutorials for estimating epigenetic age can be found in Horvath $^{2}$. By definition, IEAA is independent of blood immune cell counts (naive $\mathrm{CD}^{+} \mathrm{T}$ cells, exhausted $\mathrm{CD} 8^{+} \mathrm{T}$ cells, plasma $\mathrm{B}$ cells, $\mathrm{CD} 4^{+} \mathrm{T}$ cells, natural killer cells, monocytes, and granulocytes) estimated from DNA methylation data. EEAA is calculated using the following three steps. First, we calculated the DNAm age estimate from Hannum et al. ${ }^{1}$. Second, we increased the contribution of age related blood cell types to the DNAm age estimate by forming a weighted average of Hannum's DNAm age estimate with the following 3 blood cell type estimate: naive $\left(\mathrm{CD} 45 \mathrm{RA}^{+} \mathrm{CCR} 7^{+}\right)$cytotoxic T cells, exhausted $\left(\mathrm{CD} 28^{-} \mathrm{CD} 45 \mathrm{RA}^{-}\right)$cytotoxic $\mathrm{T}$ cells, and plasmablasts using the Klemera-Doubal approach ${ }^{48}$. The weights used in the averaging procedure were chosen on the basis of the WHI data, i.e., the same weights were used in all data sets. EEAA is positively associated with exhausted $\mathrm{CD}^{+} \mathrm{T}$ cells, plasmablast cells, and negatively associated with naive $\mathrm{CD} 8^{+} \mathrm{T}$ cells. Thus, EEAA tracks both age related changes in blood cell composition and cellintrinsic DNAm changes. Both the intrinsic and extrinsic DNAm age measures were correlated highly with chronological age within each contributing cohort $(0.63 \leq r \leq 0.97$, Supplementary Table 1$)$, except for the two Lothian birth cohorts whose participants were born in one of two single years and hence had a small age range at testing. Conversely, by definition, our measures of DNAm age acceleration, IEAA and EEAA, are not associated with chronological age.

The measures of epigenetic age acceleration are implemented in our freely available software (https://dnamage.genetics.ucla.edu) ${ }^{2}$.

Estimating blood cell counts based on DNA methylation levels. The blood cell abundance measures were estimated using two different methods. The proportions of cytotoxic $\left(\mathrm{CD}^{+}\right) \mathrm{T}$ cells, helper $\left(\mathrm{CD} 4^{+}\right) \mathrm{T}$, natural killer, B cells, and granulocytes were estimated with the Houseman method ${ }^{49}$. The percentage of exhausted $\mathrm{CD}^{+} \mathrm{T}$ cells (defined as CD28 ${ }^{-} \mathrm{CD} 45 \mathrm{RA}^{-}$), the number (count) of naive CD8 ${ }^{+}$ $\mathrm{T}$ cells (defined as $\mathrm{CD} 45 \mathrm{RA}^{+} \mathrm{CCR} 7^{+}$), and plasma blasts were estimated with the Horvath method ${ }^{14}$. These DNAm based estimates of blood cell counts are highly correlated with corresponding flow cytometric measures ${ }^{50}$.

Heritability analysis. Both IEAA and EEAA measures were adjusted for sex prior to estimation of heritability. We conducted heritability analysis using three approaches: (1) SOLAR (Sequential Oligogenic Linkage Analysis Routine) ${ }^{51}$ based on pedigree data, (2) LDSC (LD score regression) ${ }^{30}$ based on GWAS summary data, and GCTA (Genome-wide Complex Trait Analysis) ${ }^{52}$ based on SNP array data to estimate narrow sense $h^{2}$. For the Framingham heart pedigree offspring cohort, we used the additive polygenic model implemented in SOLAR to estimate the heritability of IEAA and EEAA. In this additive polygenic model, heritability is defined as the proportion of phenotypic variance attributable to genetic variation. 
Table 4 Genetic correlations with and causal effects of other complex traits on epigenetic age acceleration

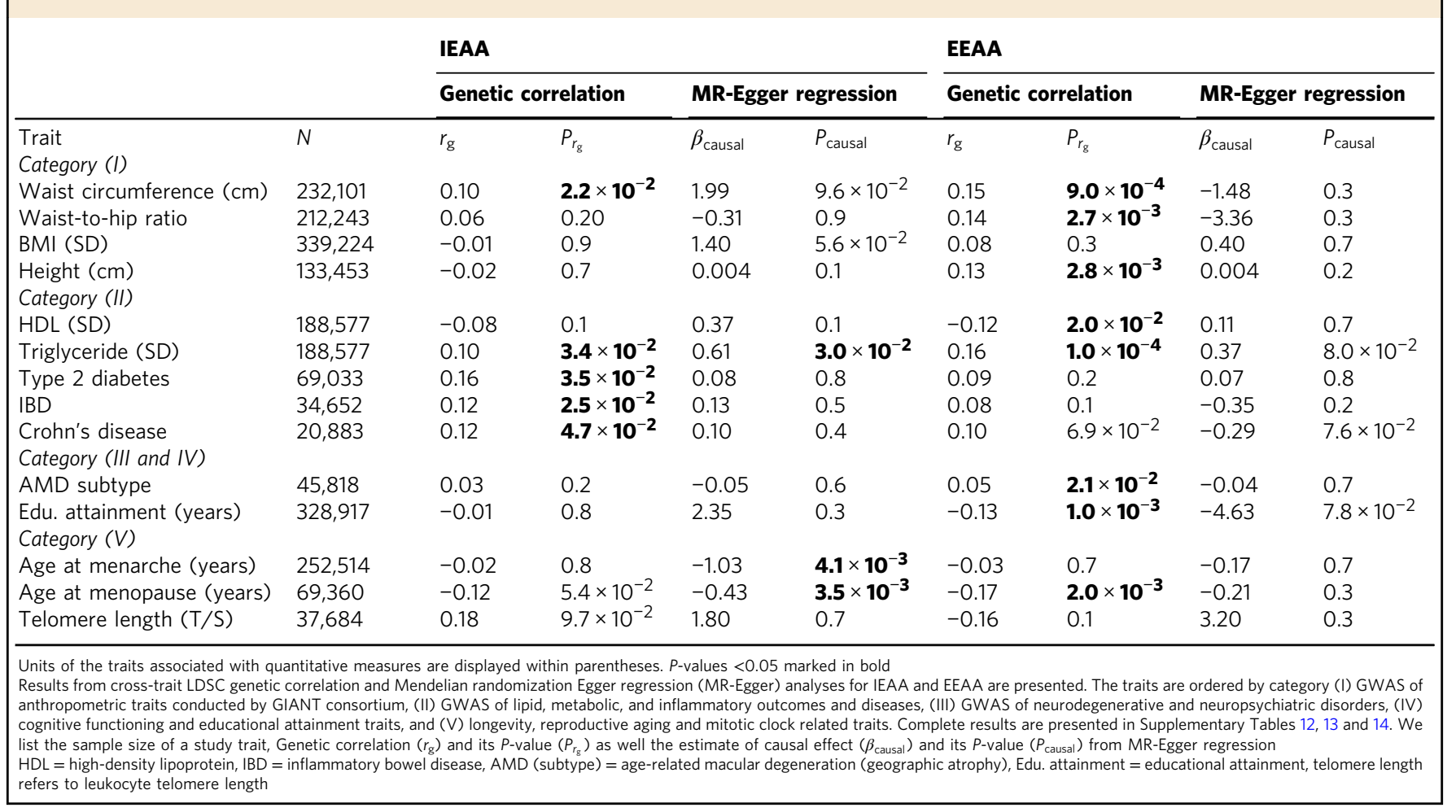

To estimate the heritability of IEAA and EEAA using the meta-analysis results from all European individuals from stage 1 (Fig. 1a) (studies 1-11, N=8393), we used LDSC analysis because it only requires GWAS summary statistics rather than genotype type data. We chose the following options for LD score regression: 1000 Genome European data (phase I) downloaded from LDSC for specifying independent variables (--ref-ld flag) and weights (--w-ld flag) in the regression. Prior to estimation, we filtered our markers to HapMap3 markers as downloaded and suggested by LDSC, which could help align allele codes of our GWAS results with other GWAS results for the genetic correlation analysis conducted later.

GCTA-REML analysis was used to estimate the heritability of IEAA and EEAA among the WHI participants of African (AFR) or Hispanic ancestry. The WHI sub-studies were genotyped on different platforms (Supplementary Table 2). In order to combine the genotype data across the studies from WHI EMPC and WHI BA23, we converted the MaCH dosage format into PLINK format and used both genotyped and imputed markers in the analysis. We only used SNP markers that could be found in all studies. We focused on high quality SNPs defined based on minor allele frequency (MAF) $>0.05$, Hardy-Weinberg equilibrium (HWE) $P$ $>0.0001$, and $\mathrm{MaCH} r^{2}>0.8$, yielding $\sim 4$ million markers. In particular, we increased the threshold of MAF to $10 \%$ in estimating the heritability of the Hispanic group in order to ensure the convergence of the REML estimation procedure. All analyses were adjusted for 4 principal components (PC).

GWAS meta-analysis. Our GWAS meta-analysis involved $~ 7.4$ million SNPs or INDEL variants, which were genotyped and imputed markers with the 1000 genomes haplotype reference panel. Prior to imputation, SNP quality was assessed by MAF, HWE, and missingness rates across individuals (Supplementary Table 4). The individual studies used IMPUTE $2^{53}$ with haplotypes phased using SHAPEIT ${ }^{54}$ or $\mathrm{MaCH}^{55}$ phased using Beagle ${ }^{56}$ or Minimac ${ }^{53}$ to impute SNP and INDEL markers based on the 1000 Genomes haplotypes released in 2011 June or 2012 March. The quality of imputed markers was assessed by the Info measure $>0.4$ (in IMPUTE2) or $R^{2}>0.3$ (in Minimac), and HWE $P>1.0 \times 10^{-6}$. To increase resolution for SNP association, a few genomic regions in the FHS cohort were also imputed based the Haplotype Reference Consortium $(N=64,976)^{57}$. FHS used linear mixed models to account for pedigree structure via a kinship matrix, as implemented in R "lmekin" package. The BSGS cohort used Merlin/QTDT ${ }^{58}$ for family-based association analysis. For other association analyses, we regressed the age acceleration trait values on estimated genotype dosage (counts of test alleles) or expected genotype dosage, implemented in Mach2QTL ${ }^{59}$, SNPTEST ${ }^{60}$, and PLINK. All association models were adjusted for sex, to account for the higher epigenetic age acceleration in men than women ${ }^{50}$, and also for PCs as needed. We included variants with $\mathrm{MAF} \geq 2 \%$. SNPs were removed from an individual study if they exhibited extreme effects (absolute regression coefficient $\beta>30$, Supplementary Table 4).
We divided the meta-analysis into two since IEAA and EEAA differ across racial/ethnic groups ${ }^{50}$. In one arm, we performed GWAS meta-analysis of IEAA and EEAA, focusing on individuals of European ancestry $(N=8393$, studies $1-11$ in Supplementary Table 1). We required a marker present in at least 5 study data sets and combined the coefficient estimates $\beta$ from each study using a fixed-effects meta-analysis model weighted by inverse variance, as implemented in the software $\mathrm{Metal}^{61}$. In the other arm, each SNP with suggestive association $\left(P<1.0 \times 10^{-5}\right)$ in Europeans was subsequently evaluated in individuals of non-European ancestry $(N$ = 1514, studies 12-15 in Supplementary Table 1). A further meta-analysis combined the GWAS findings from the two ancestries. We removed SNPs from the meta-analysis if they exhibited highly significant heterogeneity across studies (Cochran Q $I^{2} P$-value $\leq 0.001$ ), or co-located with CpG from the DNAm age predictors according to the Illumina annotation file for the Illumina Infinium $450 \mathrm{~K}$ array. We analyzed additional SNPs across all study sets to arrive at summary statistics at the combined stage, which were needed for our summary statistics based Mendelian randomization analyses. The quality of SNPs was also assessed using the Cochran Q $I^{2} P$-value.

Linkage disequilibrium analysis. Regional SNP association results were visualized with the software LocusZoom ${ }^{62}$. All linkage disequilibrium (LD) estimates presented in this article were calculated using individuals of European ancestry from the 1000 genomes reference panel (released in Oct 2012).

Conditional analyses. The conditional analysis implemented in GCTA software ${ }^{52}$ was used to test whether a given genetic locus harbored multiple independent causal variants. We conditioned on the leading SNP with the most significant metaanalysis $P$-value (Table 1). As reference panel for inferring the $\mathrm{LD}$ pattern we used the $N=379$ individuals with European ancestry from the 1000 genomes panel released in December 2013. We defined a SNP as having an additional association if it remained significant $\left(P<5 \times 10^{-8}\right)$ after conditioning on the leading SNP and also met the additional criterion $\log _{10} \mathrm{BF} \geq 6$ for a significant trans-ethnic association.

Chromatin state annotations. For each leading SNP of a significant locus, we used the UCSC genome browser to display the primary chromatin states across 127 cell/ tissue lines at $200 \mathrm{bp}$ resolution (Supplementary Fig. 8). The $n=127$ diverse cell or tissue lines were profiled by the NIH RoadMap Epigenomics ${ }^{22}(n=111)$ and ENCODE projects $^{63}(n=16)$. We used a 15-state chromatin model (from ChromHMM) which is based on five histone modification marks ${ }^{22}$.

Annotations for genome-wide significant variants. We used the HaploReg (version 4.1) tool $^{64}$ to display characters of genome-wide significant variants 
including conserved regions by GERP and SiPhy scores, DNase tracks, involved proteins and motifs, GWAS hits listed in NHGRI/EBI and functional annotation listed in dbSNP database, as summarized in Supplementary Data 1.

Leukocyte cis-eQTL analyses. To evaluate cis-eQTL in blood, our cis-eQTL study leveraged a large-scale blood expression data $(n=15,295)$ that came from five broad categories of data. The first category involved a large-scale eQTL analysis in 5257 individuals collected from the FHS pedigree cohort (of European ancestry) ${ }^{65}$. Linear mixed models were performed for the eQTL analysis, adjusted for family structure via random effects and adjusted gender, age, blood cell counts, PCs, and other potential confounders via fixed effects. The analysis was carried out using the "pedigreemm" package of R. The second category involved the significant cis-eQTL, released from GTEx (version 6 in 2015) ${ }^{66}$. The expression data from GTEx involve multiple tissues from 449 individuals of mostly $(>80 \%)$ European ancestry. We used the cis-eQTL results evaluated in 338 blood samples. The downloaded ciseQTL results only list significant results (FDR $q<0.05$ ), according to a permutation test based threshold that corrected for multiple comparisons across genes and tissue types. The third category involved the cis-eQTL results from LSMeta ${ }^{67}$, which was a large-scale eQTL meta-analysis in 5331 blood samples collected from 7 studies including our study cohort inChianti. We downloaded the cis-eQTL results from http://genenetwork.nl/bloodeqtlbrowser/. The fourth and fifth categories were discovery and replication samples from an eQTL analysis in peripheral blood $^{68}$, respectively. The publicly released cis-eQTL results only involved the 9640 most significant (FDR $q<0.01$ ) cis-eQTL results (corresponding to 9640 significant genes) from the discovery sample. The fourth category involved the expression data of 2494 twins from the NTR cohort. The fifth category involved 1895 unrelated individuals from the NESDA cohort.

For all five categories of blood data, the cis-window surrounding each SNP marker was defined as $\pm 1 \mathrm{Mb}$. We defined a significant cis-eQTL relationship by imposing the following criteria: (a) FDR $q<0.05$ for categories $1-3$, (b) FDR $q<$ 0.01 for categories 4 and 5 .

SMR analysis. $\mathrm{SMR}^{23}$ uses SNPs as instrumental variables to test for a direct association between an exposure variable and an outcome variable, irrespective of potential confounders. Unlike conventional Mendelian randomization analysis, the SMR test uses summary-level data for both SNP-exposure and SNP-outcome that can come from different GWAS studies ${ }^{23}$. We tested the expression levels of the eleven candidate genes identified in our leukocyte cis-eQTL analysis.

SMR defines a pleiotropic association as association between gene expression and a test trait due to pleiotropy or causality (Supplementary Fig. 12a, b). A significant SMR test $P$-value does not necessarily mean that gene expression and the trait are affected by the same underlying causal variant, as the association could possibly be due to the top associated cis-eQTL being in LD with two distinct causal variants. Zhu et al. ${ }^{23}$ define the scenario of several causal variants, which is of less biological interest than pleiotropy, as "linkage" and proposed a statistical test "HEIDI" for distinguishing it from pleiotropy. The null hypothesis of the HEIDI test corresponds to desirable causal scenarios. Thus, a non-significant $P$-value (defined here as $P \geq 0.01$ ) of the HEIDI test is a desirable finding. Conversely, a significant HEIDI test $P$-value indicates that at least two linked causal variants affect both gene expression and epigenetic age acceleration (Supplementary Fig. 12c).

To test the association of a given gene expression with age acceleration, we used summary level cis-eQTL results from (1) FHS, (2) GTEx, and (3) LSMeta (total: $N$ $=10,906)$.

We included the $c i s$-SNPs (with MAF $\geq 0.10$ ) within a test gene $( \pm 1 \mathrm{Mb})$. We selected the cis-SNPs as instrumental markers as follows: cis-eQTL FDR $<.05$ for GTEx, cis-eQTL $P=1 \times 10^{-6}$ for the two large-scale studies: LSMeta and FHS. All significant SNP-gene pairs were subjected to the HEIDI analysis. The analysis involves the summary data of the cis-SNPs surrounding the instrumental markers and the LD pattern evaluated from a reference panel. We used the 1000 genome individuals with ancestry of European $(N=379)$ released in December 2013 as the reference panel, imposed an LD threshold of 0.9 and selected a default setting based on a chi-square $\left(\chi_{1}^{2}\right)$ test statistic threshold of 10 for SNP pruning. The GWAS summary data were based on the meta-analysis results at the combined stage. As a sensitivity check, we repeated the HEIDI analysis using the summary GWAS data of individuals with European ancestry (studies 1-11).

Mendelian randomization analysis for LTL and IEAA. We gathered the summary statistics from three large-scale meta-analysis studies for association with LTL, including (I) the association results of 484 SNPs located in TERT locus from the study conducted by Bojesen et al. ${ }^{25}$ ( $N=53,724$ individuals of European ancestry), (II) the GWAS summary data from the study conducted by Codd et al..$^{26}(N=$ 37,684 individuals of European ancestry) downloaded from the European Network for Genetic and Genomic Epidemiology consortium (ENGAGE, see URL) and (III) the association results of 4 SNPs at $P<5.0 \times 10^{-8}$ and 1 SNPs at $P<1.0 \times 10^{-6}$ listed in Table 1 from the study conducted by Pooley et al. ${ }^{27}(N=26,089$ individuals of European ancestry). In the first study, the effect sizes were available for change in telomere length $(\Delta \mathrm{TL}>0$ indicating a test allele associated with longer LTL) and fold change in telomere length ( $>1$ indicating a test allele associated with longer LTL). We used the effect sizes with respect to $\Delta \mathrm{TL}$ in our analysis. The other two studies reported $\Delta \mathrm{TL}$ in their summary data. All telomere lengths refer to the relative telomere to single-copy gene (T/S) ratios using quantitative PCR methods with different scaling approaches applied to each study. The summary data of the second were used for bidirectional Mendelian randomization analysis. Summary statistics of IEAA and EEAA were based on the association results from the combined meta-analysis. Our SMR analysis used the summary data from studies I and II. To compare the patterns between LTL associations and IEAA associations at 5p15.33 TERT locus, we used the dense panel of SNP association results from study I (484 SNPs), as depicted in Fig. 3b.

IEAA adjusted LTL in 5p15.33 TERT locus. To inspect the SNPs on TERT locus affecting IEAA independent of LTL, we re-conducted the association analysis on a subset $(N=785)$ individuals from the WHI for whom both DNA methylation data and leukocyte telomere length measures were available. The study involved 457 individuals with European ancestry (from studies 3 and 5) and 328 individuals with Africans ancestry (from study 12). For each study, association tests were performed on IEAA and IEAA adjusted LTL, respectively. The results were combined by fixed-effects meta-analysis models.

Blood cell composition vs. variants in TERT. The imputed blood cell abundance measures were related to SNPs in the TERT locus using 8 studies from two cohorts: FHS (study 1) and WHI (studies 3-5 and 12-15, listed in Supplementary Table 1) involving $n=5373$ individuals. The following imputed blood cell counts were analyzed: naive $\mathrm{CD} 4^{+} \mathrm{T}$, naive $\mathrm{CD} 8^{+} \mathrm{T}$, exhausted $\mathrm{CD} 8^{+} \mathrm{T}$ cells (defined as $\mathrm{CD} 28$ negative CD45R-negative), plasmablasts, $\mathrm{CD}^{+} \mathrm{T}$, nature killer cells, monocytes, and granulocytes. Blood cell proportions $\left(\mathrm{CD}^{+} \mathrm{T}\right.$ cells, $\mathrm{CD} 4^{+} \mathrm{T}$ cells, NK cells, $\mathrm{B}$ cells, and granulocytes) were estimated using Houseman's estimation method which is based on DNA methylation signatures from purified leukocyte samples ${ }^{49}$ The percentage of exhausted $\mathrm{CD}^{+} \mathrm{T}$ cells (defined as $\mathrm{CD} 28^{-} \mathrm{CD} 45 \mathrm{RA}{ }^{-} \mathrm{B}$ cells) and the number of naive $\mathrm{CD} 8^{+} \mathrm{T}$ cells (defined as $\mathrm{CD} 45 \mathrm{RA}{ }^{+} \mathrm{CCR} 7^{+} \mathrm{B}$ cells) were estimated using the advanced analysis option of the epigenetic clock software ${ }^{14}$. To avoid confounding, the cell abundance measures were adjusted for chronological age by forming residuals. For each study, the SNP association analysis was performed using the same SNP makers from our genome-wide meta-analysis of IEAA and EEAA. The results were combined across studies using the same fixed-effects meta-analysis model.

In vitro studies of hTERT in human fibroblasts. Foreskins were obtained from routine elective circumcision. The tissue was cut into small pieces and digested overnight at $4{ }^{\circ} \mathrm{C}$ with Liberase, after which the epidermis was peeled away from the dermis. Several pieces of dermis were placed face down in a plastic dish with DMEM supplemented with $10 \%$ foetal calf serum, penicillin, streptomycin, and gentamycin. After incubation at $37^{\circ} \mathrm{C}$ with $5 \%$ carbon dioxide for a week, fibroblasts that emerged from the tissues were collected and expanded in fresh dishes.

Recombinant retroviruses bearing empty vector (pBabePuro) or the hTERT gene; pBabePurohTERT (kind gift from Robert Weinberg) were prepared by transfecting Phoenix A cells grown in DMEM supplemented with $10 \%$ foetal calf serum, using Profection Mammalian Transfection System (Promega Cat. No: E1200). Forty-eight hours post-transfection, recombinant viruses in the media were collected, filtered through 0.2 micrometer filter and mixed with Polybrene (Santa Cruz Biotechnology Cat. No: 134220) to a final concentration of $8 \mu \mathrm{g} / \mathrm{ml}$. Media of primary fibroblasts were removed and replaced with the virus mix. After $24 \mathrm{~h}$ at 37 ${ }^{\circ} \mathrm{C}$ (5\% carbon dioxide), the virus mix was removed and fresh media (DMEM $+10 \%$ foetal calf serum) containing $1 \mu \mathrm{g} / \mathrm{ml}$ puromycin was added to kill uninfected cells. Puromycin selection was stopped after 4 days post-selection, when all cells in the un-infected control plates were killed. Successfully infected fibroblast were collected, counted and 200,000 were seeded into $10 \mathrm{~cm}$ cell culture plates containing DMEM supplemented with $10 \%$ foetal calf serum. When cells became confluent, they were collected by trypsinisation, counted and 200,000 were seeded in fresh $10 \mathrm{~cm}$ plates. This cycle of culture, collecting and seeding was repeated until the control cells senesced. The cell numbers were used to calculate population doubling according to the following formula: Population doubling $=3.32[\log ($ final cell number) - $\log ($ number of cell seeded)]. In parallel to the proliferating culture of cells described above, separate plates of infected fibroblasts (pBabePuro and pBabePurohTERT) were grown without passaging throughout the experiment, but with regular media change three times a week for the duration of the experiment. These were regarded as "static" cultures as they were not passed since being seeded from the start of the experiment. DNA from all the collected cells were extracted according to the procedure described in the QIAamp DNA Mini Kit (Qiagen Cat. No. 51306). The DNA was subjected to DNA methylation analyses using the Illumina 450 arrays.

GWAS based enrichment analysis with MAGENTA. We used the MAGENTA software $^{29}$ to assess whether our meta-analysis GWAS results of epigenetic age acceleration are enriched for various gene sets, e.g., KEGG pathways, Gene Ontology (GO) terms, such as biological processes or molecular functions. To assign genes to SNPs, we extended gene boundaries to $\pm 50 \mathrm{~kb}$. For computational reasons, we removed categories that did not contain any genes related to age 
acceleration at a level of $P<1.0 \times 10^{-3}$ or that contained fewer than 10 genes. The cutoffs of gene set enrichment analysis (GSEA) in the MAGENTA algorithm were set at 95th and 75th percentiles which are the default parameter values for a general phenotype and for a highly polygenic trait, respectively ${ }^{29}$.

Initially, empirical $P$-values were estimated based on 10,000 permutations. For significant gene sets (empirical $P<1.0 \times 10^{-4}$ ), we estimated the final empirical $P$ value using one million permutations. We only report gene sets whose false discovery rate FDR (calculated by MAGENTA) was $<0.20$.

LDSC genetic correlation analysis. We performed cross-trait LD score regres$\operatorname{sion}^{30}$ to relate IEAA/EEAA to various complex traits (27 GWAS summary data across 23 distinct phenotypes). The GWAS results for IEAA and EEAA were based on the summary data at stage 1 analysis.

The following is a terse description of the 27 published GWAS studies. Two GWAS results in individuals of European ancestry came from the GIANT consortium on body fat distribution: waist circumference and hip to waist ratio. GWAS results of BMI and height also from the GIANT consortium. Further, we used published GWAS results from inflammatory bowel disorder (IBD) and its two subtypes: Crohn's disease and ulcerative colitis, lipid levels, metabolic outcomes and diseases: insulin and glucose levels, type 2 diabetes (stage 1 results) phenotype, age-related macular degeneration (neovascular and geographic atrophy), Alzheimer's disease (stage 1 results), attention deficit hyperactivity disorder (ADHD), bipolar disorder, major depressive disorder, schizophrenia, education attainment, age at menarche, age at menopause, LTL and longevity. The summary data of LTL was based the GWAS conducted by Codd et al. ${ }^{26}$, as described in an earlier section. A description of other published GWAS study can be found in Supplementary Note 4.

As recommend by LDSC, we filtered to HapMap3 SNPs for each GWAS summary data, which could help align allele codes of our GWAS results with other GWAS results for the genetic correlation analysis. In our GWAS studies with GCcorrection, we constrained the intercept terms with the --intercept-h2 and -intercept-gencov flags. Toward this end, we used the heritability analysis of the LDSC regression to estimate intercept terms. Additional genetic correlation analysis was performed via the LD Hub software tool ${ }^{31}$ that allowed us to analyze 233 traits. The built-in LDSC platform automatically removed all variants in the MHC region on chromosome 6 (26-34 MB).

MR-Egger regression. Under a weaker set of assumptions than typically used in Mendelian randomization (MR), an adaption of Egger regression can be used to detect and correct for the bias due to directional pleiotropy ${ }^{28}$. While the standard method of MR estimation, two-stage least squares, may be biased when directional pleiotropy is present, MR-Egger regression can provide a consistent estimate of the causal effect of an exposure (e.g., age at menopause) on an outcome trait (e.g., epigenetic age acceleration). In testing the regression model, we used the leading variants $\left(P<5.0 \times 10^{-8}\right.$ or their surrogates) from each GWAS locus associated with the exposure, as instrumental variables. We performed LD-based clumping procedure in PLINK with a threshold of $r^{2}$ set at 0.1 in a window size of $250 \mathrm{~kb}$ to yield the leading variants present in both GWAS summary data sets (for exposure and outcome), as needed. For those traits showing significant causal effects on IEAA (or EEAA), we performed a sensitivity analysis (stratified analysis) to check if the significant associations spuriously resulted from the instrumental variables (SNPs) co-locating $( \pm 1 \mathrm{Mb})$ with CpG sites from the DNAm age estimators. The random effects model meta-analysis was performed using "MendelianRandomization" R package.

GWAS-based overlap analysis. Our GWAS-based overlap analysis related gene sets found by our GWAS of epigenetic age acceleration with analogous gene sets found by published GWAS of various phenotypes. We used the MAGENTA software to calculate an overall GWAS $P$-value per gene, which is based on the most significant SNP association $P$-value within the gene boundary $( \pm 50 \mathrm{~kb})$ adjusted for gene size, number of SNPs per kb, and other potential confounders ${ }^{29}$ To assess the overlap between age acceleration and a test trait, we selected the top $2.5 \%$ (roughly 500 genes ranked by $P$-values) and top $10 \%$ genes (roughly 1900 genes) for each trait and calculated one-sided hypergeometric $P$-values ${ }^{20,69}$. In contrast with the genetic correlation analysis, GWAS based overlap analysis does not keep track of the signs of SNP allele associations.

We performed the overlap analysis for all the 23 complex traits used in the genetic correlation LDSC analysis and a few more studies that we were not able to conduct the LDSC analysis due to small sample size $(N<5000)$, negative heritability estimates or the entire study population from non-European ancestry. The additional traits included modifiers of Huntington's disease motor onset, Parkinson's disease and cognitive functioning traits (Supplementary Note 4).

URLs. URLs of databases and bioinformatics tools used throughout the masnucript are: 1000 genome project, http://www.1000genomes.org/, DNAm age, http://labs. genetics.ucla.edu/horvath/htdocs/dnamage/, ENGAGE, https://downloads.lcbru.le. ac.uk/, EIGENSTRAT, http://genepath.med.harvard.edu/ reich/Software.htm, GTEx, http://www.gtexportal.org/home/documentationPage\#AboutGTEx, GIANT, https://www.broadinstitute.org/collaboration/giant/index.php/Main_Page,
HaploReg, http://www.broadinstitute.org/mammals/haploreg/haploreg.php, HRC, http://www.sanger.ac.uk/science/collaboration/haplotype-reference-consortium, HRS, http://hrsonline.isr.umich.edu/, Illumina Infinium 450K array annotation, http://support.illumina.com/downloads/

infinium humanmethylation 450 product files.html.

IMPUTE2, https://mathgen.stats.ox.ac.uk/impute/impute_v2.html, LD score Regression, https://github.com/bulik/ldsc, LD Hub, http://ldsc.broadinstitute.org/ ldhub/, Locuszoom, http://csg.sph.umich.edu/locuszoom/, METAL, http://csg.sph umich.edu/abecasis/Metal/, MAGENTA, https://www.broadinstitute.org/mpg/ magenta/, NESDA, http://www.nesda.nl/en/, NTR, http://www.tweelingenregister. org/en/, PLINK, http://pngu.mgh.harvard.edu/ purcell/plink/, R metafor, http:// cran.r-project.org/web/packages/metafor/, R WGCNA, http://labs.genetics.ucla. edu/horvath/CoexpressionNetwork/, SHAPEIT, https://mathgen.stats.ox.ac.uk/ genetics_software/shapeit/shapeit.html, SNPTEST, https://mathgen.stats.ox.ac.uk/ genetics_software/snptest/snptest.html, YFS, http://youngfinnsstudy.utu.fi/index. $\mathrm{html}$,

Data availability. The FHS data are available at $\mathrm{dbGaP}$ under the accession numbers phs000342 and phs000724. The TwinsUK DNA methylation data are available through the NCBI Gene Expression Omnibus (GEO) under accession number GSE62992. Individual level genotype and phenotype data from TwinsUK are not permitted to be shared or deposited due to the original consent given at the time of data collection. However, genotype, DNA methylation, and phenotype data can be accessed through application to the TwinsUK data access committee (http:// www.twinsuk.ac.uk/data-access/submission-procedure/). The WHI data are available at $\mathrm{dbGaP}$ under the accession numbers phs000200.v10.p3. The phenotypes of BLSA and inChianti data are available at $\mathrm{dbGaP}$ under the accession numbers phs000215.v2.p1. LBC methylation data have been submitted to the European Genome-phenome Archive under accession number EGAS00001000910; phenotypic data are available at $\mathrm{dbGaP}$ under the accession number phs000821.v1.p1. BSGS methylation data are available from GEO accession number GSE56105. Additional information can be found in Supplementary Note 1 and Supplementary Table 3.

Received: 12 July 2017 Accepted: 19 December 2017 Published online: 26 January 2018

\section{References}

1. Hannum, G. et al. Genome-wide methylation profiles reveal quantitative views of human aging rates. Mol. Cell 49, 359-367 (2013).

2. Horvath, S. DNA methylation age of human tissues and cell types. Genome Biol. 14, R115 (2013).

3. Marioni, R. et al. DNA methylation age of blood predicts all-cause mortality in later life. Genome Biol. 16, 25 (2015).

4. Christiansen, L. et al. DNA methylation age is associated with mortality in a longitudinal Danish twin study. Aging Cell 15, 149-154 (2016).

5. Perna, L. et al. Epigenetic age acceleration predicts cancer, cardiovascular, and all-cause mortality in a German case cohort. Clin. Epigenetics 8, 1-7 (2016).

6. Chen, B. H. et al. DNA methylation-based measures of biological age: metaanalysis predicting time to death. Aging 8, 1844-1865 (2016).

7. Horvath, S. et al. Decreased epigenetic age of PBMCs from Italian semisupercentenarians and their offspring. Aging 7, 1159-1170 (2015).

8. Marioni, R. E. et al. The epigenetic clock is correlated with physical and cognitive fitness in the Lothian Birth Cohort 1936. Int. J. Epidemiol. 44, 1388-1396 (2015).

9. Levine, M. E., Lu, A. T., Bennett, D. A. \& Horvath, S. Epigenetic age of the prefrontal cortex is associated with neuritic plaques, amyloid load, and Alzheimer's disease related cognitive functioning. Aging 7, 1198-1211 (2015).

10. Horvath, S. et al. Accelerated epigenetic aging in Down syndrome. Aging Cell 14, 491-495 (2015).

11. Maierhofer, A. et al. Accelerated epigenetic aging in Werner syndrome. Aging $\mathbf{9}$, 1143-1152 (2017).

12. Horvath, S. \& Ritz, B. R. Increased epigenetic age and granulocyte counts in the blood of Parkinson's disease patients. Aging 7, 1130-1142 (2015).

13. Horvath, S. et al. Obesity accelerates epigenetic aging of human liver. Proc. Natl Acad. Sci. USA 111, 15538-15543 (2014).

14. Horvath, S. \& Levine, A. J. HIV-1 infection accelerates age according to the epigenetic clock. J. Infect. Dis. 212, 1563-1573 (2015).

15. Breitling, L. P. et al. Frailty is associated with the epigenetic clock but not with telomere length in a German cohort. Clin. Epigenetics 8, 21 (2016).

16. Levine, M. E. et al. Menopause accelerates biological aging. Proc. Natl Acad. Sci. USA 113, 9327-9332 (2016).

17. Jylhävä, J., Pedersen, N. L. \& Hägg, S. Biological age predictors. EBioMedicine 21, 29-36 (2017). 
18. Quach, A. et al. Epigenetic clock analysis of diet, exercise, education, and lifestyle factors. Aging 9, 419-446 (2017).

19. Marioni, R. E. et al. The epigenetic clock and telomere length are independently associated with chronological age and mortality. Int. J. Epidemiol. 45, 424-432 (2016).

20. Lu, A. T. et al. Genetic variants near MLST8 and DHX57 affect the epigenetic age of the cerebellum. Nat. Commun. 7, 10561 (2016).

21. Morris, A. P. Transethnic meta-analysis of genomewide association studies. Genet. Epidemiol. 35, 809-822 (2011).

22. Roadmap Epigenomics Consortium et al. Integrative analysis of 111 reference human epigenomes. Nature 518, 317-330 (2015).

23. Zhu, Z. et al. Integration of summary data from GWAS and eQTL studies predicts complex trait gene targets. Nat. Genet. 48, 481-487 (2016).

24. Day, F. R. et al. Genomic analyses identify hundreds of variants associated with age at menarche and support a role for puberty timing in cancer risk. Nat. Genet. 49, 834-841 (2017).

25. Bojesen, S. E. et al. Multiple independent variants at the TERT locus are associated with telomere length and risks of breast and ovarian cancer. Nat. Genet. 45, 371-384 (2013).

26. Codd, V. et al. Identification of seven loci affecting mean telomere length and their association with disease. Nat. Genet. 45, 422-427 (2013).

27. Pooley, K. A. et al. A genome-wide association scan (GWAS) for mean telomere length within the COGS project: identified loci show little association with hormone-related cancer risk. Hum. Mol. Genet. 22, 5056-5064 (2013).

28. Bowden, J., Davey Smith, G. \& Burgess, S. Mendelian randomization with invalid instruments: effect estimation and bias detection through Egger regression. Int. J. Epidemiol. 44, 512-525 (2015).

29. Segre, A. V., Groop, L., Mootha, V. K., Daly, M. J. \& Altshuler, D. Common inherited variation in mitochondrial genes is not enriched for associations with type 2 diabetes or related glycemic traits. PLoS Genet. 6, e1001058 (2010).

30. Bulik-Sullivan, B. et al. An atlas of genetic correlations across human diseases and traits. Nat. Genet. 47, 1236-1241 (2015).

31. Zheng, J. et al. LD Hub: a centralized database and web interface to perform LD score regression that maximizes the potential of summary level GWAS data for SNP heritability and genetic correlation analysis. Bioinformatics 33, 272-279 (2017).

32. Genetic Modifiers of Huntington's Disease Consortium. Identification of genetic factors that modify clinical onset of Huntington's disease. Cell 162, 516-526 (2015)

33. Yui, J., Chiu, C. P. \& Lansdorp, P. M. Telomerase activity in candidate stem cells from fetal liver and adult bone marrow. Blood 91, 3255-3262 (1998).

34. Gorbunova, V. \& Seluanov, A. Telomerase as a growth-promoting factor. Cell Cycle 2, 534-537 (2003).

35. Gorbunova, V., Seluanov, A. \& Pereira-Smith, O. M. Expression of human telomerase (hTERT) does not prevent stress-induced senescence in normal human fibroblasts but protects the cells from stress-induced apoptosis and necrosis. J. Biol. Chem. 277, 38540-38549 (2002).

36. Stampfer, M. R. et al. Expression of the telomerase catalytic subunit, hTERT, induces resistance to transforming growth factor $\beta$ growth inhibition in p16INK4A (-) human mammary epithelial cells. Proc. Natl Acad. Sci. USA 98, 4498-4503 (2001).

37. Young, J. I., Sedivy, J. M. \& Smith, J. R. Telomerase expression in normal human fibroblasts stabilizes DNA 5-methylcytosine transferase I. J. Biol. Chem. 278, 19904-19908 (2003).

38. Hiyama, E. \& Hiyama, K. Telomere and telomerase in stem cells. Br. J. Cancer 96, 1020-1024 (2007).

39. Simpkin, A. J. et al. Prenatal and early life influences on epigenetic age in children: a study of mother-offspring pairs from two cohort studies. Hum. Mol. Genet. 25, 191-201 (2016)

40. Simpkin, A. J. et al. The epigenetic clock and physical development during childhood and adolescence: longitudinal analysis from a UK birth cohort. Int. J. Epidemiol. 46, 546-558 (2017).

41. Chen, B. et al. Leukocyte telomere length, T cell composition and DNA methylation age. Aging 9, 1983-1995 (2017).

42. Harley, C. B. Telomere loss: mitotic clock or genetic time bomb? Mutat. Res 256, 271-282 (1991).

43. Levy, M. Z., Allsopp, R. C., Futcher, A. B., Greider, C. W. \& Harley, C. B. Telomere end-replication problem and cell aging. J. Mol. Biol. 225, 951-960 (1992).

44. Weischer, M. et al. Short telomere length, myocardial infarction, ischemic heart disease, and early death. Arterioscler. Thromb. Vasc. Biol. 32, 822-829 (2012).

45. Lowe, D., Horvath, S. \& Raj, K. Epigenetic clock analyses of cellular senescence and ageing. Oncotarget 7, 8524-8531 (2016).

46. Day, F. R., Elks, C. E., Murray, A., Ong, K. K. \& Perry, J. R. Puberty timing associated with diabetes, cardiovascular disease and also diverse health outcomes in men and women: the UK Biobank study. Sci. Rep. 5, 11208 (2015).

47. Mostafavi, $H$. et al. Identifying genetic variants that affect viability in large cohorts. PLOS Biol. 15, e2002458 (2017).
48. Klemera, P. \& Doubal, S. A new approach to the concept and computation of biological age. Mech. Ageing Dev. 127, 240-248 (2006).

49. Houseman, E. et al. DNA methylation arrays as surrogate measures of cell mixture distribution. BMC Bioinform. 13, 86 (2012).

50. Horvath, S. et al. An epigenetic clock analysis of race/ethnicity, sex, and coronary heart disease. Genome Biol. 17, 171 (2016).

51. Almasy, L. \& Blangero, J. Multipoint quantitative-trait linkage analysis in general pedigrees. Am. J. Hum. Genet. 62, 1198-1211 (1998).

52. Yang, J., Lee, S. H., Goddard, M. E. \& Visscher, P. M. GCTA: a tool for genome-wide complex trait analysis. Am. J. Human. Genet. 88, 76-82 (2011).

53. Howie, B., Fuchsberger, C., Stephens, M., Marchini, J. \& Abecasis, G. R. Fast and accurate genotype imputation in genome-wide association studies through pre-phasing. Nat. Genet. 44, 955-959 (2012).

54. O'Connell, J. et al. A general approach for haplotype phasing across the full spectrum of relatedness. PLoS Genet. 10, e1004234 (2014).

55. Li, Y., Willer, C. J., Ding, J., Scheet, P. \& Abecasis, G. R. MaCH: using sequence and genotype data to estimate haplotypes and unobserved genotypes. Genet. Epidemiol. 34, 816-834 (2010).

56. Browning, S. R. \& Browning, B. L. Rapid and accurate haplotype phasing and missing-data inference for whole-genome association studies by use of localized haplotype clustering. Am. J. Hum. Genet. 81, 1084-1097 (2007).

57. McCarthy, S. et al. A reference panel of 64,976 haplotypes for genotype imputation. Nat. Genet. 48, 1279-1283 (2016).

58. Abecasis, G. R., Cardon, L. R. \& Cookson, W. O. A general test of association for quantitative traits in nuclear families. Am. J. Hum. Genet. 66, 279-292 (2000).

59. Li, Y., Willer, C., Sanna, S. \& Abecasis, G. Genotype imputation. Annu. Rev. Genom. Hum. Genet. 10, 387-406 (2009).

60. Marchini, J. \& Howie, B. Genotype imputation for genome-wide association studies. Nat. Rev. Genet. 11, 499-511 (2010).

61. Willer, C. J., Li, Y. \& Abecasis, G. R. METAL: fast and efficient meta-analysis of genomewide association scans. Bioinformatics 26, 2190-2191 (2010).

62. Pruim, R. J. et al. LocusZoom: regional visualization of genome-wide association scan results. Bioinformatics 26, 2336-2337 (2010).

63. EncodeProject. An integrated encyclopedia of DNA elements in the human genome. Nature 489, 57-74 (2012).

64. Ward, L. D. \& Kellis, M. HaploReg: a resource for exploring chromatin states, conservation, and regulatory motif alterations within sets of genetically linked variants. Nucleic Acids Res. 40, D930-D934 (2012).

65. Joehanes, R. et al. Integrated genome-wide analysis of expression quantitative trait loci aids interpretation of genomic association studies. Genome Biol. 18, 16 (2017).

66. The GTEx Consortium. The Genotype-Tissue Expression (GTEx) pilot analysis: multitissue gene regulation in humans. Science 348, 648-660 (2015).

67. Westra, H.-J. et al. Systematic identification of trans eQTLs as putative drivers of known disease associations. Nat. Genet. 45, 1238-1243 (2013).

68. Wright, F. A. et al. Heritability and genomics of gene expression in peripheral blood. Nat. Genet. 46, 430-437 (2014).

69. $\mathrm{Lu}, \mathrm{A}$. T. et al. Genetic architecture of epigenetic and neuronal ageing rates in human brain regions. Nat. Commun. 8, 15353 (2017).

\section{Acknowledgements}

The study was supported by U34AG051425-01, NIA/NIH 5R01AG042511-02, and the Paul G. Allen Frontiers Group. The WHI program is funded by the NIH/NHLBI, U.S Department of Health and Human Services through contracts NIH/NHLBI 60442456 BAA23 (Assimes, Absher, Horvath), HHSN268201100046C,HHSN268201100001C, HHSN268201100002C, HHSN268201100003C, HHSN268201100004C, and HHSN271201100004C, Epigenetic Mechanisms of PM-Mediated CVD Risk (WHIEMPC), NIH/NIEHS R01ES020836 (Whitsel, Baccarelli; Hou). SNP Health Association Resource project (WHI-SHARe), NIH/NHLBI N02HL64278 GWAS of Hormone Treatment and CVD and Metabolic Outcomes within the Genomics and Randomized Trials Network" (WHI-GARNET), NIH/NHGRI U01HG005152 (Reiner). The Framingham Heart Study is funded by the National Institutes of Health contract to Boston University N01-HC-5195 and HHSN268201500001I and its contract with Affymetrix, Inc for genotyping services (Contract No. N02-HL-6-4278). Funding to support the Omni cohort recruitment, retention, and examination was provided by the NHLBI Contract N01-HC-25195 and HHSN268201500001I, as well as NHLBI grants R01HL070100, R01-HL076784, R01-HL-49869, and U01-HL-053941.” The DNA methylation resource from the FHS was funded by the Division of Intramural Research, National Heart, Lung, and Blood Institute, National Institutes of Health. L.X., K.L.L., and J.M.M. were supported by R01AG029451. D.P.K. was supported by R01AR041398 and R01AR061162. TwinsUK is funded by the Wellcome Trust and European Community's Seventh Framework Programme (FP7/2007-2013), and also receives support from the National Institute for Health Research (NIHR)-funded BioResource, Clinical Research Facility and Biomedical Research Centre based at Guy's and St Thomas' NHS Foundation Trust in partnership with King's College London. SNP Genotyping in TwinsUK was 
performed by The Wellcome Trust Sanger Institute and National Eye Institute via NIH/ CIDR. P.-C.T. and J.T.B. were supported by ESRC (ES/N000404/1). EPIC-Norfolk is supported by the Medical Research Council (MRC) [G9502233; G0401527; G100143; MC_PC_13048; MR/L00002/1] and Cancer Research UK [C864/A8257]. A.C., F.R.D., N. J.W., J.R.B.P., and K.K.O. are supported by the MRC [MC_UU_12015/1;

MC_UU_12015/2].

\section{Author contributions}

A.T.L. carried out all of the non-study-specific statistical analyses and wrote the first draft of the article. L.X. performed most FHS specific analyses and preliminary meta-analyses. K.R. performed the in vitro experiments. All authors participated in the interpretation of the study and helped revise the article. S.H. conceived the study.

\section{Additional information}

Supplementary Information accompanies this paper at https://doi.org/10.1038/s41467017-02697-5.

Competing interests: The authors declare no competing financial interests.

Reprints and permission information is available online at http://npg.nature.com/ reprintsandpermissions/
Publisher's note: Springer Nature remains neutral with regard to jurisdictional claims in published maps and institutional affiliations.

\begin{abstract}
(c) (i)
Open Access This article is licensed under a Creative Commons Attribution 4.0 International License, which permits use, sharing, adaptation, distribution and reproduction in any medium or format, as long as you give appropriate credit to the original author(s) and the source, provide a link to the Creative Commons license, and indicate if changes were made. The images or other third party material in this article are included in the article's Creative Commons license, unless indicated otherwise in a credit line to the material. If material is not included in the article's Creative Commons license and your intended use is not permitted by statutory regulation or exceeds the permitted use, you will need to obtain permission directly from the copyright holder. To view a copy of this license, visit http://creativecommons.org/ licenses/by/4.0/.
\end{abstract}

(C) The Author(s) 2018

\section{Ake T. Lu', Luting Xue ${ }^{2}{ }^{2}$, Elias L. Salfati ${ }^{3}$, Brian H. Chen $\left(^{4,5}\right.$, Luigi Ferrucci ${ }^{4}$, Daniel Levy ${ }^{5}$, Roby Joehanes $\left(^{5}{ }^{5}\right.$, Joanne M. Murabito ${ }^{6}$, Douglas P. Kiel ${ }^{7}$, Pei-Chien Tsai ${ }^{8}$, Idil Yet ${ }^{8}$, Jordana T. Bell ${ }^{8}$, Massimo Mangino ${ }^{8}$, Toshiko Tanaka ${ }^{4}$, Allan F. McRae $\odot^{9,10}$, Riccardo E. Marioni ${ }^{9,11,12}$, Peter M. Visscher $0^{9,10}$, Naomi R. Wray $0^{9,10}$, Ian J. Deary ${ }^{11}$, Morgan E. Levine', Austin Quach', Themistocles Assimes ${ }^{3}$, Philip S. Tsao ${ }^{3,13}$, Devin Absher ${ }^{14}$, James D. Stewart ${ }^{15}$, Yun Li ${ }^{16,17}$, Alex P. Reiner ${ }^{18}$, Lifang Hou ${ }^{19,20}$, Andrea A. Baccarelli ${ }^{21}$, Eric A. Whitsel $\oplus^{15,22}$, Abraham Aviv" ${ }^{23}$, Alexia Cardona ${ }^{24}$, Felix R. Day $\circledast^{24}$, Nicholas J. Wareham ${ }^{24}$, John R.B. Perry ${ }^{24}$, Ken K. Ong ${ }^{24,25}$, Kenneth Raj ${ }^{26}$, Kathryn L. Lunetta ${ }^{2} \&$ Steve Horvath ${ }^{1,27}$}

\footnotetext{
'Human Genetics, David Geffen School of Medicine, University of California, Los Angeles, Los Angeles, CA 90095, USA. ${ }^{2}$ Department of Biostatistics, Boston University School of Public Health, Boston, MA 02118, USA. ${ }^{3}$ Department of Medicine, Stanford University School of Medicine, Stanford, CA 94305, USA. ${ }^{4}$ Intramural Research Program, National Institute on Aging, National Institutes of Health, Baltimore, MD 21224, USA. ${ }^{5}$ National Heart, Lung and Blood Institute, Bethesda, MD 20824-0105, USA. ${ }^{6}$ Department of Medicine, Section of General Medicine, Boston University School of Medicine, Boston, MA 02118, USA. ${ }^{7}$ Institute for Aging Research, Hebrew SeniorLife, Beth Israel Deaconess Medical Centre, Harvard Medical School, Boston, MA 02215, USA. ${ }^{8}$ Department of Twin Research and Genetic Epidemiology, Kings College London, London SE1 7EH, UK. ${ }^{9}$ Institute for Molecular Bioscience, The University of Queensland, Brisbane 4072 QLD, Australia. ${ }^{10}$ Queensland Brain Institute, The University of Queensland, Brisbane 4072 QLD, Australia. " Centre for Cognitive Aging and Cognitive Epidemiology, Department of Psychology, University of Edinburgh, 7 George Square, Edinburgh EH8 9JZ, UK. ${ }^{12}$ Medical Genetics Section, Centre for Genomic and Experimental Medicine, Institute of Genetics and Molecular Medicine, University of Edinburgh, Edinburgh EH4 2XU, UK. ${ }^{13}$ VA Palo Alto Health Care System, Palo Alto, CA 94304, USA. ${ }^{14}$ HudsonAlpha Institute for Biotechnology, Huntsville, AL 35806, USA. ${ }^{15}$ Department of Epidemiology, Gillings School of Global Public Health, University of North Carolina, Chapel Hill, NC 27599, USA. ${ }^{16}$ Department of Genetics, School of Medicine, University of North Carolina, Chapel Hill, NC 27599, USA. ${ }^{17}$ Department of Biostatistics, Gillings School of Global Public Health, University of North Carolina, Chapel Hill, NC 27599, USA. ${ }^{18}$ Fred Hutchinson Cancer Research Center Box 358080, WHI Clinical Coordinating Ctr/Public Health Sciences M3-A4, Seattle, WA 98109, USA. ${ }^{19}$ Department of Preventive Medicine, Feinberg School of Medicine, Northwestern University Chicago, Evanston, IL 60611, USA. ${ }^{20}$ Center for Population Epigenetics, Robert H. Lurie Comprehensive Cancer Center, Feinberg School of Medicine, Northwestern University Chicago, Evanston, IL 60611, USA. ${ }^{21}$ Laboratory of Environmental Epigenetics, Departments of Environmental Health Sciences Epidemiology, Columbia University Mailman School of Public Health, New York, NY 10032, USA. ${ }^{22}$ Department of Medicine, School of Medicine, University of North Carolina, Chapel Hill, NC 27516, USA. ${ }^{23}$ The Center for Human Development and Aging, University of Medicine and Dentistry, New Jersey Medical School, Rutgers, Newark, NJ 07103, USA. ${ }^{24}$ Medical Research Council (MRC) Epidemiology Unit, University of Cambridge School of Clinical Medicine, Institute of Metabolic Science, Cambridge Biomedical Campus, Cambridge CB2 OSL, UK. ${ }^{25}$ Department of Paediatrics, University of Cambridge School of Clinical Medicine, Cambridge Biomedical Campus, Cambridge CB2 OSP, UK. ${ }^{26}$ Radiation Effects Department, Centre for Radiation, Chemical and Environmental Hazards, Public Health England, Chilton, Didcot, Oxfordshire OX11 ORQ, UK. ${ }^{27}$ Biostatistics, School of Public Health, University of California, Los Angeles, Los Angeles, CA 90095, USA. Ake T. Lu and Luting Xue contributed equally to this work. Ken K. Ong, Kenneth Raj, Kathryn L. Lunetta, and Steve Horvath jointly supervised this work.
} 